\title{
TRACKING INFLUENZA IMMUNIZATION \\ IN THE COMMUNITY
}

\section{CENTRE IOOR NEWIOUNDLAND STUDIES}

TOTAL OF 10 PAGES ONLY MAY BE XEROXED 



National Library

of Canada

Acquisitions and

Bibliographic Services

395 Wellington Street Ottawa ON K1A ON4

Canada
Bibliothèque nationale

du Canada

Acquisisitons et services bibliographiques

395 , rue Wellington Ottawa ON K1A 0N4

Canada
Your file Votre référence ISBN: 0-612-93051-3 Our file Notre référence ISBN: 0-612-93051-3
The author has granted a nonexclusive licence allowing the National Library of Canada to reproduce, loan, distribute or sell copies of this thesis in microform, paper or electronic formats.

The author retains ownership of the copyright in this thesis. Neither the thesis nor substantial extracts from it may be printed or otherwise reproduced without the author's permission.
L'auteur a accordé une licence non exclusive permettant à la Bibliothèque nationale du Canada de reproduire, prêter, distribuer ou vendre des copies de cette thèse sous la forme de microfiche/film, de reproduction sur papier ou sur format électronique.

L'auteur conserve la propriété du droit d'auteur qui protège cette thèse. $\mathrm{Ni}$ la thèse ni des extraits substantiels de celle-ci ne doivent être imprimés ou aturement reproduits sans son autorisation.
In compliance with the Canadian

Privacy Act some supporting forms may have been removed from this dissertation.

While these forms may be included in the document page count, their removal does not represent any loss of content from the dissertation.
Conformément à la loi canadienne sur la protection de la vie privée, quelques formulaires secondaires ont été enlevés de ce manuscrit.

Bien que ces formulaires aient inclus dans la pagination, il n'y aura aucun contenu manquant. 



\title{
TRACKING INFLUENZA IMMUNIZATION IN THE COMMUNITY
}

\author{
by \\ CSuzette Porter \\ A thesis submitted to the \\ School of Graduate Studies \\ in partial fulfillment of the \\ requirements for the degree of \\ Master of Nursing \\ School of Nursing \\ Memorial University of Newfoundland
}

May 2003

St. John's

Newfoundland 


\begin{abstract}
Objective: To evaluate and compare the effectiveness of using three different influenza immunization surveillance forms in accounting for all influenza vaccine distributed in a community setting. Methods: All family practice physicians and community health nurses (CHNs) in the Eastern Region of Newfoundland and Labrador were randomly sent one of three influenza immunization surveillance forms: individual, tally or government. They were asked to: document case information about patients given influenza vaccine in the 2001-2002 season, return completed forms as well as unused vaccine, and provide feedback about the forms at the end of the study. Results: There was $100 \%$ participation by CHNs and $82.4 \%$ by physicians. Of the 13,310 doses of influenza vaccine distributed, $7,645(57.5 \%)$ doses were accounted for using the surveillance forms. Use of the government form accounted for statistically significantly more influenza vaccine than use of the tally and individual forms ( $p<0.0005)$, while the tally form accounted for significantly more vaccine than the individual form $(\mathrm{p}<0.0005)$. The tally and individual forms provided more information than the government form about those who received the vaccine. Feedback identified that health care professionals preferred less paper and fewer questions, but supported influenza immunization surveillance. Conclusion: The study's findings supports using a tally form as a way to collect useful influenza immunization surveillance data in future influenza immunization seasons.
\end{abstract}




\section{Acknowledgments}

I wish to thank those who provided support throughout the many phases of this work over the past two years. First of all I would like to thank Dr. Donna Moralejo, my thesis advisor, for her time, patience and support. Without your knowledge and guidance I would have never been able to complete this document.

I would also like to thank Dr. Catherine Donovan, for being a member of my thesis committee and providing me with her support and expertise throughout this study. Thank you also for advocating on my behalf to help me obtain in kind support from Health and Community Services Eastern Board to complete this study.

I wish to thank the ARNNL research and trust for their financial support and Health and Community Services Eastern Board for providing some in kind support which helped in the completion of this work. I would also like to thank my colleges of Health and Community Services, especially those in the Holyrood office, for all their support over the past two years.

To my parents, Eva and Norman Spurrell, thank you for supporting me through everything that I have ever done, and for being there whenever I need you. Because of you I have learned from an early age that hard work does indeed pay off, and that I can do anything that I "put my mind to". I love you both very much.

Finally, to my dearest husband Neil, you were the inspiration for the completion of my Masters Degree. How can I ever thank you for all the support you have given me throughout this entire process. You were always there to tell me that I would get through 
it, especially during the challenging times. I will never forget all the little things that you have done for me to help me complete my degree, like driving me to class and the library during snow storms. You are the best! Thank you so much. I love you. 


\section{Table of Contents}

Abstract ii

Acknowledgments

List of Tables viii

List of Figures $\quad$ ix

Chapter

1 INTRODUCTION 1

Research Questions $\quad 5$

2 LITERATURE REVIEW 6

Influenza $\quad 6$

Impact of Influenza $\quad 7$

Effectiveness of Influenza Immunization 15

Effectiveness of Influenza Immunization Campaigns 21

The Need for Influenza Immunization Surveillance 24

Methods of Influenza Immunization Surveillance in 25

the Community

Nurses and Influenza Immunization Surveillance 32

Evaluating Surveillance Systems 34

Summary $\quad 36$

3 METHODOLOGY $\quad 39$

Design of Study and Surveillance Initiative 39

Setting and Respondents $\quad 40$

Surveillance Forms $\quad 40$

Data Collection Procedure: Immunization Surveillance 41

Data Collection: Evaluation of Surveillance Forms 43

Data Analysis $\quad 43$

Ethical Considerations $\quad 44$ 
Participation $\quad 47$

Distribution and Administration of Influenza Vaccine $\quad 49$

Recipient Profile $\quad 54$

Completeness of Data $\quad 59$

Participant Feedback $\quad 62$

Feedback From Other Provincial Regions 68

Target Groups Vaccinated $\quad 69$

$\begin{array}{ll}\text { Summary } & 70\end{array}$

$\begin{array}{lll}5 & \text { DISCUSSION } & 74\end{array}$

Evaluation of Surveillance Forms and Systems 74

Overall Sensitivity $\quad 77$

Sensitivity by Surveillance Form $\quad 82$

Vaccine Wastage $\quad 83$

Summary of Sensitivity $\quad 84$

Positive Predictive Value $\quad 86$

Simplicity and Acceptability $\quad 87$

Complete, Accurate and Useful Data 91

Flexibility $\quad 97$

Representativeness 98

Timeliness 103

Conclusions Regarding Improving Influenza 104

Immunization Surveillance

Improving Surveillance of Target Groups $\quad 107$

Improving Vaccination of Target Groups 109

Strengths of the Study 111

Limitations of the Study $\quad 112$

6 IMPLICATIONS FOR PRACTICE, POLICY, 113 EDUCATION, AND RESEARCH

Summary of Discussion of Study Findings 113

Implications for Community Health Practice 116

$\begin{array}{ll}\text { Implications for Policy } & 118\end{array}$

Implications for Education $\quad 120$

Implications for Research $\quad 121$

$\begin{array}{ll}\text { Overall Conclusions } & 123\end{array}$ 
APPENDIX A: Logic Model 


\section{List of Tables}

Table 1 Distribution of Forms Between Participating 48 Physician and All Physicians Who Were Originally Distributed Forms

Table 2 Participation and Nonparticipation of Physicians in Small and Large Clinics by Form Type

Table 3 Influenza Vaccine Distributed, Administered and Administered + Returned by Health Care

Professionals Using the Three Surveillance Forms

Table 4 Reasons Individuals Received Influenza Vaccine Indicated By Health Care Professionals Who Completed the Tally and Individual Forms

Table 5 Proportion of Data Missing for Each Question by Tally and Individual Forms

Table 6

Proportion of Missing Data for each Question by Physician and Community Health Nurse 


\section{List of Figures}

Figure 1 Proportion of Influenza Vaccine Doses Distributed, Administered and Administered + Returned Using the Three Surveillance Forms 


\section{CHAPTER 1}

\section{Introduction}

Influenza is an acute respiratory illness which occurs worldwide and causes considerable morbidity and mortality each year. It is estimated that each year in Canada up to 75,000 hospitalizations and 6700 deaths are associated with influenza virus infection (Health Canada, 1993). Vaccination against the influenza virus is recognized to be the single most effective way of preventing or attenuating influenza for those at high risk of serious illness or death (Health Canada, 2000a). The groups most susceptible to complications related to influenza are: persons 65 years of age or older, persons with chronic cardiac or pulmonary disorders or other chronic conditions, residents of institutions, and children and adolescents with conditions treated for long periods of time with acetylsalicylic acid. The National Advisory Committee on Immunization (NACI) has identified these high risk groups, and health care professionals who could transfer influenza to these populations, as targets for annual immunization programs (Health Canada, 2000a).

Realistically, influenza immunization programs should aim to vaccinate at least 90\% of eligible recipients (Health Canada, 2000a). Currently, influenza vaccination rates of eligible groups are much lower than the target rate of $90 \%$, since vaccination rates of residents living in long term care facilities is only $70 \%$, and only $20 \%$ to $40 \%$ of adults and children with medical conditions that leave them susceptible to complications related 
to influenza, receive the vaccine annually (Duclos, Arruda, \& Dessau, 1996; Dewals, Carbonneau, \& Payette, 1996). Studies of health care workers in hospitals and long term care facilities have shown influenza vaccination rates of $26 \%$ to $61 \%$ (Potter, Stott, \& Roberts, 1999; Wilde, McMillan, \& Serwint, 1999; Nichol \& Hauge, 1997). It is important to increase vaccination rates in order to decrease the impact of influenza and to help in preparing for pandemic influenza.

Pandemic influenza planning is ongoing due to the threat of a predicted worldwide outbreak. This threat has created an interest in increasing influenza vaccine coverage rates as well as a need for more accurate influenza immunization data. It is therefore appropriate that officials in Newfoundland and Labrador implement a mandatory influenza immunization surveillance program to track influenza immunization.

It is important for nurses and other health care professionals to enhance influenza vaccination promotion campaigns since annual fall campaigns have been helpful in increasing influenza vaccination coverage rates (Health Canada, 1993). The proportion of vaccine eligible groups receiving influenza vaccination must be determined in order to decide who the health promotion campaign should target. To calculate the proportion of vaccine eligible groups that receive influenza vaccine, the number of individuals vaccinated (numerator) is required, as well as the number of individuals eligible (denominator). A surveillance system to account for the number of influenza vaccine doses administered, and to whom they are administered, will provide numerator data, 
while denominator data may be available from other areas, such as census data and disease registries.

Prior to the 2001-2002 season, in Newfoundland and Labrador, there was no influenza immunization surveillance system in place to determine the numerator data. Therefore this study implemented a surveillance system to account for the influenza vaccine distributed and administered. If the appropriate denominator data is available, a surveillance system will provide information about those who receive the influenza vaccine, so that health care professionals can focus influenza immunization promotion campaigns on target groups who fail to receive influenza vaccination. This should consequently lead to an increased influenza vaccination rate in eligible groups. A graphical representation of this process can be found in appendix A.

Currently in Newfoundland and Labrador, the publically funded influenza immunization program covers those individuals identified by the NACI as high risk for complications as well as health care workers and essential service workers. Each year, vaccine is distributed across the province to public health units, nurses, physicians' offices, long term care facilities, and hospitals. In the 2001-2002 season there were approximately 86,000 doses of influenza vaccine distributed across the province. The vaccine distributed, however, provides only a crude measure of the vaccination rate since the number of doses distributed does not equate to the actual number of doses administered, nor does it identify whether the vaccine was administered to the appropriate people. 
In Newfoundland and Labrador, prior to the 2001-2002 season, there was no system available to track the number of doses of influenza vaccine administered in either the hospital or community settings. The number of doses of influenza vaccine administered in an institutional setting is much easier to determine compared to those in a community setting, since this data can be extracted from patient files or occupational health files. The only way the number of doses of influenza vaccine administered in a community setting had been determined was through counting the doses of vaccine distributed minus the doses of vaccine returned. This is not an accurate measure because many health care professionals fail to return unused vaccine (personal communication, Patricia Heath, Communicable Disease Control Coordinator Eastern Region, November $24,2002)$. It was therefore important to develop a surveillance system which would provide valid and reliable data and meet the seven criteria identified by the Centres for Disease Control (CDC) (2001) which are important to consider when evaluating a surveillance system. The seven criteria which are necessary for an efficient and effective system are: adequate sensitivity, adequate positive predictive value, simplicity, acceptability, flexibility, representativeness, and timeliness.

In the 2001-2002 season a surveillance system was developed using one of three influenza immunization surveillance forms as a way to account for all influenza vaccines distributed in a community setting. Two of the surveillance forms, individual and tally forms, contained exactly the same information, but were presented in different formats. Heath care providers who completed the individual or tally form were required to provide 
information on six items for each individual who received the vaccine. The third form, the government form, was sent out by the provincial government and only required the health care professional to indicate whether or not the individual was under or over 65 years of age. This study evaluated the three surveillance forms according to the seven criteria identified by the CDC (2001) to determine which form was most effective in providing information about the influenza doses distributed during the 2001-2002 season. This study also provided community health nurses and physicians with an opportunity to provide feedback about surveillance and the influenza immunization surveillance forms used in this study.

\section{Research Questions}

The research questions are:

1. Which surveillance form (tally form, individual form, or government form) is the most sensitive in providing information about the number of doses of influenza vaccine administered and to whom?

2 Which surveillance form provides the most complete and accurate data?

3. Which surveillance form provides the most useful information about those who receive influenza vaccine?

4. Which surveillance form is more acceptable to health care professionals?

5. What factors influence health care professionals' participation in influenza immunization surveillance? 


\section{CHAPTER 2}

\section{Literature Review}

The literature review provides an overview of the influenza virus, and it's impact, and the effectiveness of influenza vaccine in preventing and attenuating the influenza virus in different population groups. A review of studies conducted on influenza immunization surveillance, and the forms used, as well as how to effectively evaluate a surveillance system, will follow.

\section{Influenza}

Influenza $A$ and $B$ are the two main types of influenza viruses. Influenza $A$ viruses, which undergo antigenic changes every year, are the most common cause of epidemic influenza (Health Canada, 2000b). Influenza B viruses are more localized and may be restricted to one country or region in any given year (Health Canada, 2000b).

The influenza virus is a complex and dynamic virus. Influenza A viruses are classified into subtypes based on their hemagglutinin $(\mathrm{H})$ and neuraminidase $(\mathrm{N})$ antigens. Antibodies to these antigens, particularly to $\mathrm{H}$ antigen, can protect an individual from a virus carrying the same antigen. During inter-pandemic periods, minor $\mathrm{H}$ antigen changes or "drift" are common. It is this antigenic variation from one influenza virus subtype to another that is responsible for continued outbreaks of influenza and that necessitates annual reformulation and administration of the influenza vaccine (Health 
Canada 2002). The antigens of influenza $B$ viruses are much more stable than those of influenza A viruses and, although antigenic variation does occur, it is less frequent. The influenza virus may also undergo a major antigenic change or "shift" with different $H$ and often a different $\mathrm{N}$ antigen from strains circulating previously (Health Canada, 2002). This may result in pandemic influenza, meaning worldwide epidemic.

Influenza $\mathrm{A}$ and $\mathrm{B}$ are viruses that exist worldwide. The occurrence of influenza is not bound to seasons, and can occur all year round especially in tropical climates. In temperate zones, influenza is predominately a winter disease. In Canada, there may be outbreaks of influenza anytime during the fall and winter with definite outbreaks being common from November or December through April and May. According to Health Canada (2000b) there is no specific reason identified as to why influenza is predominately a winter disease. However, it is likely that the transmission of the virus is facilitated by people congregating indoors more during the winter, or drier indoor air which may help the virus survive longer. This also may be seen in the tropical zones of the world where the incidence of influenza is increased during the monsoons or rainy season which may generate indoor crowding (Kilbourne, 1987).

\section{Impact of Influenza}

Influenza is an acute disease of the respiratory tract that typically has a sudden onset with headache, chills, and cough followed by fever, loss of appetite, muscle aches, and extreme fatigue (Chin, 2000). Most people recover completely from influenza 
within one to two weeks, however, severe complications can occur particularly in children, elderly people, and individuals with chronic conditions such as cardiac and pulmonary conditions.

Several studies have examined the relationship between the presence of circulating influenza virus and hospital admissions of individuals over age 65 years. Upshur, Knight, and Goel (1999) conducted a time series analysis from 1988 to 1993 to determine the relationship between influenza virus and hospital admissions of the elderly in Ontario, for pneumonia, chronic lung disease, and congestive heart failure. To perform the time series analysis, auto regressive integrated moving average transfer function models were used. These models were compared with simple cross correlations by using Pearson's product moment correlation. The results showed a statistically significant correlation between the presence of influenza virus and admissions of the elderly for pneumonia in all five years of the study.

The study by Upshur et al. (1999) was subject to several limitations. The data used was administrative data collected by a records clerk when the patient was discharged. This data was not collected specifically for research purposes, therefore, the applicability of the data may be questioned. Another limitation of the database was the chance of multiple re-admissions which would increase the numerator and, therefore, bias the rates to overestimate the impact of influenza. There was also a possibility of misclassification of the diagnosis because up to 15 diagnostic codes may have been entered on any one patient. This could have caused confusion around the diagnosis most 
responsible for the admission.

Another study conducted by the Manitoba Centre for Health Policy and Information (2001) determined hospital influenza pressure periods each year from 19951999. The weekly average number of hospital emergency room visits, visits to doctors, and drug prescriptions for influenza related illness were calculated. For the 1998-1999 season, ambulatory visits for influenza like illness went from 7,821 per week in the noninfluenza period to 10,984 visits per week in the influenza period, which showed a $40 \%$ increase. At the Health Sciences and St. Boniface hospitals in Winnipeg, emergency room visits for influenza like illness went from 176 to 282 visits per week, showing an increase of $60 \%$ during the influenza period. The number of antibiotics prescribed per week increased $40 \%$ when the influenza virus was circulating. By examining these indicators, it was also found that more than half $(54 \%)$ of the patients admitted to hospital for influenza like illness were aged 65 years and older.

Two recent studies showed high influenza associated morbidity in healthy young children. Neuzil, Mellen, Wright, Mitchel, and Griffin (2000) showed that the rate of hospitalizations associated with influenza in infants younger than six months was similar to that of adults at high risk of influenza (104 excess cases per 10,000). Izurieta, Thompson and Kramarz (2000) studied hospitalization rates for acute respiratory disease among infants and young children younger than two years of age who did not have conditions that put them at high risk for complications of influenza. The hospitalization rates were 231 per 100,000 person-months for one site and 193 per 100,000 person- 
months for the other site. These rates were 12 times higher than rates among older children, age 5 to 17 years, without high risk conditions.

The validity of the conclusions of the studies by Neuzil et al. (2000) and Izurieta et al. (2000) depends on the ability to distinguish the effect of the influenza virus from that of other infections that produce respiratory illness during the winter. Due to the expensive nature of testing patients for the presence of specific viruses, both studies used records of respiratory illnesses in hospitals and clinics for the defined population. Data obtained from viral surveillance systems were used to define the timing of the influenza epidemics. This information was then used to assess the burden of influenza virus infection in various populations. Both studies tried to separate influenza virus from other viruses that are prevalent during the winter months. According to Gruber (1998) in most years even when the influenza virus is circulating, more children are hospitalized with respiratory syncytial virus (RSV) than with influenza. Because Izurieta et al. used hospital and clinic records as a way to distinguish respiratory virus infections from influenza, rather than testing the patients, this method may not have been a very effective one. Izurieta et al. defined periods when influenza predominated as all periods of two or more weeks in which at least $5 \%$ of the total number of influenza virus isolates and less than $5 \%$ positive tests for RSV were seen. If there were any periods where the actual number of RSV infections in a given age group was large in relation to the number of influenza infections, influenza might not have been dominant during some of those periods. Also, the comparisons were done in the summer and parts of the winter without 
known RSV activity, so the potential effects of undetected RSV remains a problem. These studies introduce uncertainty about whether influenza is responsible for all, or even most of the excess morbidity that is attributed to it, but the overall evidence does suggest that influenza is indeed a problem.

In Canada, the age standardized rate for hospitalizations for those 65 years of age and older due to influenza and pneumonia in the year of 1997-1998 was 1241 per 100,000 population (Canadian Institute for Health Information, 1999). In Newfoundland for the year of 1997-1998, the age standardized rate for hospitalization was 1319 per 100,000 population.

The influenza virus does not only have an impact on increasing hospitalization rates but it is also associated with an increase in mortality. Influenza and pneumonia together are the sixth leading cause of death in Canada (Statistics Canada, 1997). The most recent calculated age standardized mortality rate due to pneumonia and influenza was in 1997 which showed a rate of 24.0 per 100,000 (95\% CI: 23.0-23.6) (Statistics Canada, 1997). It is important to note that this mortality rate does not separate influenza and pneumonia, and it does not separate cases of pneumonia that are secondary to influenza from other cases of pneumonia. The age standardized mortality rate due to influenza and pneumonia has been consistent over the previous four years. In 1993, the age standardized mortality rate for influenza and pneumonia was the same as it was in 1997. In Newfoundland, the age standardized mortality rate for influenza and pneumonia in 1996 was 21.2 per 100,000 (95\% CI:18.9- 23.5) (Statistics Canada, 1996). 
While cancer has the highest age standardized mortality rate of 181.5 per 100,000 population, influenza and pneumonia have a higher age standardized mortality rate than diabetes which has an age standardized mortality rate of 17.4 per 100,000 population. Influenza and pneumonia also have a higher age standardized mortality rate than eight of the other selected leading causes of death which include: hereditary and degenerative diseases of central nervous system, suicide, nephritis, chronic liver disease, psychiatric disorders and HIV infection (Statistics Canada, 1997).

The crude mortality rate in Canada for the year of 1997 from influenza and complications as a result of the influenza virus is 2.0 per 100,000 population. This can be calculated with Canada having a midyear population in 1997 of $29,987,200$ and a total of 608 influenza related deaths (Statistics Canada, 1997). With such a small crude mortality rate it can be seen that deaths due to influenza are not the main reason why influenza is being addressed at the population level.

Influenza is also of concern because it has an impact on health care expenditures. Health Canada (1998) determined the economic burden of illnesses in Canada. Influenza and pneumonia accounted for 386.1 million dollars of health care expenditures in Canada for 1998. When comparing the economic burden of influenza and pneumonia to other illnesses, breast cancer accounted for 84.8 million dollars, lung cancer for 227.5 million dollars, and diabetes for 203.5 million dollars of the health care expenditures in 1998. Thus influenza and pneumonia had the greatest economic impact.

The influenza virus also has an effect on school and work absenteeism. Neuzil, 
Hohlbein, and Zhu (2002) conducted a one year prospective study to quantify the effect of the influenza season on illness episodes, school absenteeism, medication use, parental absenteeism from work, and the occurrence of secondary illness in families among a cohort of children enrolled in an elementary school during the 2000-2001 influenza season. Of the 428 families with 611 children enrolled in the school, 216 families with 313 children chose to participate in the study. The parent or guardian of a child participating in the study was sent a survey when their child was absent from school. This survey was sent to parents to determine the reason why the child was absent. The results of the study showed that during the influenza season the number of illness episodes were 1.5 times more likely, school absenteeism was 1.7 times more likely, and parental work absenteeism was 1.6 times more likely to occur. In addition, the number of household members ill in three days after the child was absent from school was 2.2 times more during the influenza season.

The results of this study by Neuzil et al. (2002) were based on the surveys they received and not on laboratory or clinical evaluation. It was assumed that non-influenza illnesses occurred equally during the influenza and non-influenza winter season, therefore, any excess illness during the influenza season was attributable to influenza infection. Influenza infection actually may not have been the child's diagnosis which may have altered the results. The rates of parental absenteeism could have been higher because the rates presented in this study were based solely on missing work to care for a sick child. These rates did not include work that the parent may have missed if the child 
transmitted influenza to the parent.

The discussion of influenza's impact on society would not be complete without some mention of influenza pandemics. Pandemic influenza, meaning worldwide epidemic, is usually associated with a major antigenic change or "shift" and the rapid global spread of influenza $\mathrm{A}$ virus with $\mathrm{H}$ and often a different $\mathrm{N}$ antigen from strains circulating previously (Health Canada, 2002). Due to the fact that the virus undergoes an antigenic shift, everyone is susceptible to infection and will be at greater risk of developing severe complications due to influenza infection (Kilbourne, 1987). The vaccine takes time to be produced to protect against this particular strain and is therefore not immediately available to protect against the new strain of influenza.

In the past century there have been three pandemics. The influenza pandemic of 1918, known as the "Spanish flu,"was the most deadly pandemic in history, killing 20 million people, primarily young adults (Kilbourne, 1987). Although mortality rates associated with more recent pandemics of 1957, known as the "Asian flu", and of 1968, known as the "Hong Kong flu,"were reduced in part by antibiotic therapy for secondary bacterial infections and more aggressive supportive care, both were associated with high rates of morbidity and social disruption (Kilbourne, 1987). The CDC (2001a) predicts that the next pandemic will infect up to 200 million people in the US alone. Of those 200 million people affected, 88,000 to 300,000 persons will die. Depending on the virulence and penetrance of infection, pandemic influenza could result in as many as 51,000 deaths in Canada (Tam, 1999). In 1997, the number of influenza related deaths in Canada was 
calculated to be 608 . When comparing the projected 51,000 deaths due to influenza and related complications that could occur during the next pandemic to the 608 deaths that were seen in 1997, the potential impact that future influenza pandemics may have on morbidity and mortality can be easily seen.

The warning period preceding spread of the pandemic strain in Canada is likely to be relatively short, therefore, vaccine will have to be distributed and administered as rapidly as possible. It is important to have mechanisms in place, far in advance of the pandemic, so that vaccine supplies can be efficiently distributed and high rates of compliance achieved. There will be no time available to change attitudes and convince individuals to receive the vaccine. Therefore, during the inter-pandemic period, it is important to achieve high influenza immunization rates so individuals will be used to receiving the vaccine and will therefore be willing to receive it during a pandemic.

In summary, although it is difficult to completely separate influenza from other respiratory infections, the influenza virus does increase morbidity and mortality, health care expenditures and absenteeism during the influenza season. Therefore, prevention of influenza and its complications is warranted.

\section{Effectiveness of Influenza Immunization}

The influenza vaccine is recognized to be the single most effective way of preventing influenza for those at high risk of serious illness or death (Health Canada, 2000a). The influenza vaccines presently used in Canada are inactivated suspensions of 
one or more strains of virus grown in hens' eggs with thimersol as a preservative and possibly gelatin as a stabilizer (Health Canada, 1998). The virus strains chosen for inclusion in influenza vaccine are reviewed annually to ensure they include antigens that are expected to provide the best protection against the strains anticipated to be circulating during the following winter. Protection of the vaccine usually begins two weeks after injection and may last for six months or longer.

Numerous studies have demonstrated the effectiveness of influenza immunization. Several retrospective studies suggest that vaccination of elderly people results in a decrease in complication rate by $70 \%$, and mortality by $87 \%$ (Barker $\&$ Mullooly, 1980; Barker \& Mullooly,1981; \& Barker \& Mullooly, 1982). Govaert, Sprenger, Dianant, Aretz, Masurel, and Knotterus (1994) conducted a prospective randomized double blind placebo-controlled trial to determine the efficacy of influenza vaccine in elderly individuals. There were a total of 1838 participants in the study aged 60 years or older and both randomly assigned groups were similar with respect to age, sex, risk factors, previous vaccination in past two years, and protective antibody titre before vaccination. The placebo group $(n=911)$ received an intramuscular placebo containing physiological saline solution and the experimental group $(n=927)$ received the influenza vaccine composed in accordance with the advice of the World Health Organization. The main outcome measures were: the identification of influenza like illness up to five months after vaccination; self reported influenza through postal questionnaires at ten weeks and five months after vaccination, and serological influenza 
(which was defined as a serum sample with a four fold increase of antibody titre between three weeks and five months after vaccination). The vaccine group was noted to have a lower incidence of serological influenza (4\%), than the placebo group (9\%) (RR: .5; $95 \%$ CI: .35 to .61$)$. The incidence of clinical influenza was also lower in the vaccine group (2\%), in comparison to $3 \%$ in the placebo group (RR: .53; $95 \%$ CI: .39 to .73 ). The effect was strongest for the combination of both serological and clinical influenza (RR : .42; $95 \%$ CI: .23 to .74$)$. This study shows that influenza vaccination may halve the incidence of serological and clinical influenza in the elderly.

Influenza infection is known to be more severe in patients with chronic renal diseases, therefore, it is recommended that these patients receive annual influenza immunization (CDC, 1998). However, there are some discrepancies between the results of studies on immune response to influenza vaccination in patients with renal disease. Some of these studies confirmed a good seroresponse in this group of patients, while others indicated significantly lower antibody levels compared with healthy people (Descamps-Latscha \& Chatenoud, 1996; Steele, 1994). Because of mild or severe immunological disorders observed in patients with renal disorders, a poorer immune response would normally be expected in this group (Descamps-Latscha \& Chatenoud, 1996). However, 22 children with nephrotic syndrome achieved a four-fold titre increase which ranged, depending on the vaccine strain, from $68 \%$ to $86 \%$ after influenza vaccination. This was comparable to the response rates seen in healthy people (Brydak, Rajkowski \& Machala, 1998). 
Furth, Neu, and McColley (1995) conducted a study on children with end stage renal disease requiring dialysis and on patients after renal transplantation. This study showed that these patients benefited from vaccination against influenza, regardless of the severity of renal disease. This study also calculated a protection rate, defined as the proportion of subjects exceeding the threshold titre of 100 after vaccination. Protection rates depending on the vaccine strain, ranged from $66 \%$ to $80 \%$ in children with chronic renal failure, $80 \%$ to $100 \%$ in dialysed patients, and $71 \%$ to $86 \%$ in the healthy group four weeks after vaccination. Convincing results were also obtained by Grekas, Alivanis, and Kotzadamis (1992) where the percentage of 19 patients on haemodialysis (age 20 to 60 years) showing at least a four-fold titre increase which ranged, depending on the vaccine strain, from $31.5 \%$ to $47 \%$ one month after vaccination and $87.5 \%$ to $100 \%$ two months after vaccination.

Individuals with diabetes have been identified by the National Advisory Committee on Immunization as a group of individuals at increased risk for developing complications due to influenza infection. Diepersloot, Bouter, and Beyer (1987) conducted a study on 159 patients with type 1 or type 2 diabetes who were vaccinated with whole virus influenza vaccine. The humoral immune response was lower in patients with diabetes compared with healthy vaccinated controls, however, the difference was not statistically significant between these two groups. The protection rates measured after vaccination ranged, depending on the vaccine strain, from $57 \%$ to $85 \%$ in the patients with diabetes and $50 \%$ to $90 \%$ in the control groups. The protection 
rates were between $44 \%$ and $78 \%$ in patients with type 1 diabetes and between $65 \%$ and $87 \%$ in patients with type 2 , while in the control groups these values were from 67 to $100 \%$.

Influenza vaccine is also effective in reducing mortality during an epidemic, as can be seen by the study conducted by Ahmed, Nicholson and Nguyen-Van-Tam (1995). Their case control study assessed the effectiveness of influenza vaccine in reducing mortality during an epidemic. Subjects were 16 years of age or older who died between November 4, 1989 and February 23, 1990. General practitioners' reports were reviewed for 315 patients who had died of influenza during an epidemic and 777 controls who died from a cause other than influenza a year after the epidemic. Cases and controls were matched for age, sex, existence of chronic illness, and area of residence. Conditional logistic regression analysis for matched case-control studies showed that influenza vaccination reduced mortality by $41 \%(95 \% \mathrm{CI}: 13$ to 60$)$ for all subjects. There was no statistically significant difference in the effect of vaccine between subjects who lived in institutions and in the community $(p=0.16)$, or between subjects with high risk medical conditions and those without $(\mathrm{p}=0.76)$.

Based on studies among young healthy volunteers, influenza vaccination appears to be $40 \%$ to $70 \%$ protective (McDonald \& Andrews, 1955 ; Members of the Commission on Influenza, 1953 and Committee on Clinical Trials of Influenza Vaccine, 1953). Although vaccination in healthy adults is protective, the influenza immunization program for this population has been scrutinized in terms of its cost effectiveness. 
Nichol (2001) assessed the economic implications of a strategy for annual influenza vaccination of healthy adults age 18 to 64 years. The cost benefit analysis included direct and indirect costs associated with vaccination, as well as costs saved by vaccination. Clinical and economic variable estimates were derived primarily from published literature. Monte Carlo simulation was used to calculate the mean net costs or savings, along with the $95 \%$ probability interval, and sensitivity analyses explored the sensitivity of the cost model to different values of the input variables. The study showed an average annual savings of $\$ 13.66$ per person vaccinated. Seventy eight percent of costs savings were costs related to lost work productivity. In Newfoundland, there were 86,000 doses of influenza vaccine distributed last year. Assuming that the annual savings per person vaccinated were the same in Newfoundland as those in the study by Nichol and the number of influenza vaccine doses distributed $(86,000)$ were equal to the number administered, there would be a potential cost savings of $\$ 1,174,760$ per population vaccinated. This cost savings calculation may be a gros overestimation because a large portion of the $(86,000)$ doses of vaccine may have been administered to elderly individuals who do not work, while Nichol's calculation was based on lost productivity. The findings of the study by Nichol are valid only if the underlying assumptions and values used for the individual variables included in the model are valid. The model assumed that the persons targeted for vaccination were full time, year round workers, therefore the findings may not apply to part time, seasonal workers. There were no studies found that were conducted on the cost savings for older individuals. 


\section{Effectiveness of Influenza Immunization Campaigns}

Influenza immunization programs are presently not reaching the target goal of immunizing $90 \%$ of those who should receive the vaccine. Because influenza immunization is known to significantly decrease rates of influenza, it is important to understand why individuals are not receiving influenza vaccine and to determine ways to promote influenza vaccination.

Yassi, Murdazk, Cheang, Tran and Aoki (1994) used a self-administered questionnaire to determine the knowledge, attitude, and behaviour regarding influenza vaccine of 519 health workers from high risk hospital areas. There were 948 questionnaires sent with a response rate of $55 \%$. There was no statistically significant differences noted between the demographic profiles of responders and non-responders. The knowledge of the health care workers regarding influenza vaccine was assessed: $50 \%$ knew who should receive the vaccine, $56 \%$ knew the influenza vaccine was important for health care workers as they could easily spread it to patients. Registered nurses had more knowledge regarding influenza vaccine than other occupations. A positive correlation was seen between greater knowledge of vaccine and willingness to recommend it to others. However, there was no correlation between knowledge and willingness to receive vaccine, as nurses were noted to have the most knowledge but highest refusal rate of $80.5 \%$. Health care professionals in this study were most willing to receive vaccine under certain conditions: if they heard about the vaccine from their private physicians; if the vaccine was offered to them; and if they had the vaccine in the 
past and experienced only a few post-vaccine side effects. This study suggests that knowledge does not change behaviour, however, strong promotional efforts and easy onsite access may help increase influenza immunization rates.

The study by Yassi et al. (1994) showed that there was no correlation between knowledge and willingness to receive influenza immunization. Well structured influenza immunization programs have been used as a method to increase vaccine uptake. A cross sectional survey conducted by McArthur, Simor, Campbell, and McGeer (1999) consisted of mailing 1,520 questionnaires to long term care facilities for the elderly in Canada. They looked at which vaccination programs were associated with high resident vaccination rates. The overall survey response rate was $1,270(84 \%)$, of which the mean overall vaccination rate was $79 \%$. By using multi-variable analysis, increased vaccination rates were associated with several variables; vaccine being offered to all residents rather than some, having a single non-physician staff member organize the program, and obtaining consent for immunization upon admission rather than annually. Although each component was associated with a modest increase in rates, the mean vaccination rate was $40 \%$ in facilities with one of the factors previously mentioned, and $91 \%$ in those with all of the above components.

McAuther et al. (1999) found that well organized influenza vaccination programs increase the influenza vaccination rates of residents in Canadian long term care facilities. In addition to well structured influenza immunization programs, strong influenza immunization promotional campaigns can also increase influenza 
immunization rates. Ontario's universal immunization program, which was initiated in the 2000-2001 influenza season, is an example of an immunization program that used a strong promotional campaign. This program consisted of publicly funded influenza vaccine for all individuals as well as strategies to increase vaccination rates and decrease rates of influenza. According to Ontario's Ministry of Health and Long Term Care (2001) the promotional campaigns included use of communication products such as letters to the health care community, public and businesses. There were community outreach activities such as local media events, public health unit driven events and clinics, a toll free number with information about the program, targeted information kits for public health units, doctors, hospitals, and employers, and website pages. The awareness of the program increased by $41 \%$ from July to December 2000 , and at the end of the campaign only $9 \%$ of individuals were not able to identify a specific component of the program. There were 7.9 million doses of vaccine distributed for the universal influenza program.

As a way to begin to evaluate Ontario's universal influenza immunization program one can compare the number of laboratory confirmed cases of influenza in Ontario in the 1999-2000 to the 2000-2001 season. In the 1999-2000 season up to and including the week ending April 14, 2000 there were 2,889 confirmed cases of influenza. In the year of 2000-2001, Ontario's laboratory confirmed cases of influenza decreased to 840 (Ontario's Ministry of Health and Long Term Care, 2001). The number of influenza outbreaks in care facilities had also decreased from 341 in 1999- 
2000 to 7 in 2000-2001. Despite the fact that there is very little information available on the number of individuals immunized during the universal program, there was a significant decline in the number of influenza cases reported. This decrease may be due to the universal immunization program which had been initiated for that influenza season.

Reports of confirmed influenza were lower all across Canada in the 2000-2001 season (Canadian Communicable Disease Report, 2001). Therefore, it is difficult to determine if the rates of influenza have actually declined by looking at differences in the laboratory confirmed cases from year to year. Monitoring of laboratory confirmed cases of influenza is not intended to count the actual number of cases during the particular influenza season, but rather to determine where there is influenza activity. Therefore the decrease in the number of cases presented by the Ontario's Ministry of Health and Long Term care should be interpreted with caution, and a more effective method of determining influenza rates and accounting for the influenza vaccine distributed should be put in place.

\section{The Need For Influenza Immunization Surveillance}

Studies have shown that influenza immunization is effective in preventing influenza for those at high risk of serious illness. Every year, provincial community health boards distribute vaccine to physicians' clinics and public health offices. The vaccine distributed is a crude measure that is not synonymous with vaccine actually 
given. "The goal of influenza immunization programs should be $100 \%$ coverage of vaccine eligible groups to prevent serious morbidity and mortality due to influenza in both interpandemic and pandemic periods" (Health Canada, 1993, p.141). Realistically, influenza vaccination programs should aim to vaccinate at least $90 \%$ of eligible recipients (Health Canada, 2000a). It is important for health care providers to develop influenza immunization promotion campaigns, since annual fall campaigns have been helpful in increasing influenza vaccination coverage rates (Health Canada, 1993).

The proportion of vaccine eligible groups receiving influenza vaccination should be determined in order to decide who the health promotion campaign should target. To calculate the percentage of vaccine eligible groups that receive influenza vaccine, the number of individuals vaccinated (numerator) is required, as well as the number of individuals eligible (denominator). Presently, in Newfoundland and Labrador, there are no surveillance systems in place in community settings to determine if vaccine eligible individuals who reside in the community are actually receiving annual influenza vaccine. Without such a system it is difficult to determine the number of influenza immunizations administered each year and if influenza vaccination programs are reaching those who are considered to be the target group.

\section{Methods of Influenza Immunization Surveillance in the Community}

There is more documented literature about influenza immunization surveillance for institutionalized individuals than in community settings. Russell (2001) conducted a 
study to determine influenza vaccination rates in Alberta's long term care facilities. An anonymous survey was sent to Alberta nursing homes and auxiliary hospitals in the spring of 1999. Of the 160 facilities surveyed, 136 responded. Of those who responded, 85 provided data on staff vaccination rates and 118 provided data on resident vaccination rates. For institutions reporting this information, the median proportion of staff vaccinated was $29.9 \%$ and the median proportion of residents vaccinated was $91 \%$. Vaccination rates were easily determined for residents since the institutions had structured influenza vaccination programs and the programs were the responsibility of one department. Influenza vaccination records could therefore be retrieved without difficulty from residents' files. Influenza immunization surveillance is more difficult to track in the community since there are many professionals in different locations providing influenza vaccination and all community based health care providers have different recording systems.

Through searching Medline and CINAHL data bases there were only a few studies found which looked at community based influenza immunization surveillance. Several of the surveillance methods which have been tried consisted of denominator based tracking systems, random digit dialling surveys and sending out influenza immunization surveillance forms to health care providers who administer influenza vaccine.

Buffington, Bell, and Laforce (1991) conducted a study to measure the impact of a denominator based tracking system on influenza immunization rates. These 
researchers looked at 13 practices with 45 private practice physicians in the Rochester, New York area. All patients 65 years of age and older who were seen in participating physicians' practices within two years preceding the study were considered as the target population for the study. There were two intervention groups and one control group in this study. In the first intervention group, the target population for each physician (which was the denominator) was entered on a specifically designed poster. Physicians were asked to record all immunizations given to the target population and on a weekly basis tabulate a cumulative total. From this, the physicians were asked to calculate the proportion of the target population vaccinated and graph the percentage on the poster. The second intervention group did the same as the first, however, they also sent postcard reminders to all their patients aged 65 years and older. The control group did not use the poster, nor did they send out reminders, they simply determined the number of patients who were 65 years and over that had received vaccine by reviewing computer generated billing codes at the end of the study season.

The results of the study by Buffington et al. (1991) showed that the control group immunized $50 \%(2,405$ of 4,772$)$ of its target population, while the poster and poster/postcard groups immunized $66 \%(1,420$ of 2,149$)$ and $67 \%(2,427$ of 3,604$)$ respectively. The difference in the proportions immunized by all three groups was statistically significant $(\mathrm{p}<0.001)$. The goal of this study was not to determine denominator data for the purpose of influenza immunization surveillance but rather to use denominator tracking methods such as the poster and postcard reminders to increase 
influenza immunization rates in the identified target population. A denominator based tracking system could be used as a method of influenza immunization surveillance, however, it may not be a practical way to collect data on all the target groups who receive influenza immunization.

Squires, Macey, and Tam (2001) of the Division of Respiratory Diseases of Health Canada, commissioned a polling company to conduct a random-digit dialling telephone survey to estimate influenza and pneumococcal vaccine coverage rates for the 2000-2001 influenza season. Residents from all provinces and territories were included, and provincial and territorial samples were weighted based on their proportion of the Canadian population. The survey took place in January/February 2001, and 3501 non-institutionalized Canadian residents 18 years of age and older were surveyed. The results of the survey showed that $38.4 \%$ (95\% CI: $35.1 \%-41.8 \%)$ of individuals with high risk medical problems received influenza vaccine in the $2000-2001$ season. In comparison, $69.1 \%(95 \% \mathrm{CI}: 65.0 \%-73.2 \%)$ of individuals over 65 years of age received influenza vaccine. Half, $54.8 \%(95 \%$ CI: $49.0 \%-60.6 \%)$, of health care workers surveyed received vaccine.

This study shows that there is still a significant proportion of individuals with high risk conditions and health care workers who do not receive influenza vaccine. Despite the fact that this study shows the proportion of individuals age 65 years and older that receive influenza vaccine to be $69.1 \%$, there is still work needed to increase vaccination rates since the aim of influenza immunization programs is to vaccinate $90 \%$ 
of eligible recipients (Health Canada, 2000a).

The strength of the study by Squires et al. (2001) is the large sample size and the ability to get denominator data and generalize the results because they used random sampling. However, random-digit dialling telephone surveys are not a practical way to track influenza immunization in the community. This method is a very time intensive way to perform surveillance and it is not a mechanism that could be used every year to receive influenza immunization data, particularly in small regions.

Macdonald, Roberecki, and Conway (1996) examined influenza vaccine distribution and the population immunized by primary care physicians, public health nurses, and institutions, in the Rural Interlake region of Manitoba. In August of 1994, the Medical Officer of Health $(\mathrm{MOH})$ asked each provider to estimate the influenza vaccine doses required for the upcoming season. The number of doses requested were then sent to the health care professional with a survey form to record the doses as given according to the target risk group. The completed form was to be returned to the $\mathrm{MOH}$ by January 1995 . The overall survey response was $82.7 \%$. The four public health nursing offices and 16 institutions had a response of $100 \%$ and there was a response of $71.9 \%$ for the 32 participating physician offices. Of the 8,960 doses distributed, it was determined that 7,260 (77.7\%) doses were actually accounted for and given. Two thirds $(64.7 \%)$ of the vaccine accounted for was given to individuals age 65 years and older.

The use of an influenza immunization surveillance form in the study by 
Macdonald et al. (1996) was a successful mechanism to collect influenza immunization information as they were able to account for $77.7 \%$ of the vaccine distributed. The forms were returned to the $\mathrm{MOH}$ when completed. This may be a reason for their high response rate as participants may have seen it as a requirement. There was no copy provided of the form sent to health care providers in the article published by Macdonald et al. which outlined the study. The Manitoba Health department was contacted to determine if a copy of the form used in the study by Macdonald et al could be obtained. Several Medical Officers of Health were contacted but the form used in this study was not located. Therefore it was difficult to determine how the information was collected and what the form looked like. There was also no reason provided as to why $28.1 \%$ of physicians failed to respond to the survey. In addition, the researcher did not receive opinions from the respondents on the surveillance form and the form was not evaluated.

Manitoba's Communicable Disease Control Unit also developed an influenza immunization surveillance form to capture case by case information on clients receiving influenza vaccinations in the $1999-2000$ season. Prior to this, no formal tracking mechanism was in place to capture adult immunization data. Vaccine coverage had been estimated by the number of doses distributed and population estimates obtained from national studies such as population health surveys (Manitoba Health, 2000). The form consisted of a tally style form which collected the patients' names, as well as their date of birth, date of vaccination, gender, and whether they were over 65 years, or over 65 years with a chronic illness, health care staff, or "other". In total, 18,279 records 
representing data on individual patients were entered for the 1999-2000 season. The study did not reveal the number of doses distributed or the proportion of doses that were tracked. However, in the previous season there were 170,000 doses of influenza vaccine distributed. A proportion of the 18,279 records received were not from the actual forms but were obtained from physician claim data. The study failed to separate the proportion of data from the forms or physicians' claims. The forms were not sent to fee for service physicians, as they were in the study by Macdonald et al. (1996). It was felt that these physicians would not complete the forms (personal communication, Jackie Habing, Influenza Prevention Coordinator Manitoba Health, July 4, 2001).

If a similar number of influenza vaccine doses was distributed in the 1999-2000 season, the surveillance system only accounted for $10 \%$ of the doses administered. This may be due to the fact that the Communicable Disease Control unit in Manitoba did not have a formal mechanism in place to track receipt of surveillance forms and the responsibility to collect the information and forward the data on to the Communicable Disease Control unit was the responsibility of each health provider. Under reporting was known to have occurred because forms were received after the surveillance initiative was over. Surveillance was also initiated late in the campaign and several influenza vaccination clinics had already taken place before the surveillance program was endorsed by the Regional Health Authority.

In summary, the random digit dialling survey used by Squires et al. (2001) was not a practical way to collect influenza immunization surveillance data. Buffington et 
al. (1991) used a denominator based tracking system as a way to increase influenza immunization rates. This system could also be used to collect influenza immunization surveillance data, however, it would not be a practical way to collect data on all the target groups who should receive the influenza vaccine. The influenza immunization surveillance forms used in the study by Macdonald et al. (1996) and those used by Manitoba Health (2000) appear to be a more practical method of conducting influenza immunization surveillance in a community setting.

\section{Nurses and Influenza Immunization Surveillance}

Public health nurses have always played an essential role in immunization surveillance since they provide and document childhood immunizations and use this information to help plan and evaluate immunization programs (Zahner,1999). In the Eastern Region of Health and Community Services, in Newfoundland and Labrador, community health nurses administered over $80 \%$ of childhood vaccinations (personal communication, Patricia Heath, Communicable Disease Control Coordinator Eastern Region, November 24,2002 ). There is a structured mechanism in place where community health nurses send their childhood immunization surveillance forms to the communicable disease unit for data entry. This process takes place yearly and has existed for many years. The nurses are sent yearly reminders to submit their forms and there is a $100 \%$ response rate to childhood immunization surveillance. Patricia Heath (personal communication, November 24, 2002) attributes this success to the fact that 
nurses have administered the majority of childhood immunizations and are familiar with documenting and submitting this information for data entry. There is one person responsible for coordinating the submission of the forms which makes it a very structured mechanism. In addition, all the community health nurses are employees of the same Health and Community Services Board. This helps because all community health nurses follow the same guidelines and the expectation to complete surveillance forms is the same for everyone. The community health nurses are aware of the importance of collecting immunization statistics and they aim to have a $100 \%$ childhood immunization rate.

There were only a few studies found regarding nurses and their involvement with surveillance systems. The study by Macdonald et al. (1996), which was previously described, showed that the public health nursing offices' response to the influenza immunization surveillance initiative was $100 \%$, whereas the physicians' response was only $71.9 \%$. The study also found that public health nursing offices had a higher proportion, $86.9 \%$, of doses administered to doses distributed than did physicians, $75.4 \%$. This study showed that all the nurses who were sent influenza vaccine participated and had less wastage of the vaccine they had been distributed.

Nurses were involved with completing the influenza immunization surveillance forms developed and sent out by the Communicable Disease Control Unit in Manitoba in 1999-2000. Jackie Habing (personal communication, Influenza Prevention Coordinator Manitoba Health, July, 4, 2001) stated that the Communicable Disease 
Control Unit in Manitoba only sent their forms to nurses and salaried physicians.

Despite the fact that fee for service physicians received influenza vaccine, they did not send fee for service physicians their influenza immunization surveillance forms because it was felt that they would not complete the forms, since they had difficulty receiving similar information from them in the past.

Public health nurses play a significant role in immunization surveillance. They are responsible for a large percentage of childhood immunizations and have a structured mechanism in place to track these immunizations. The study by Macdonald et al. (1996) showed that nurses had a higher response rate with returning their surveillance forms and had a higher ratio of the number of doses administered to doses distributed. Public health nurses are familiar with carrying out immunization surveillance and therefore can be a great asset in collecting immunization data.

\section{Evaluating Surveillance Systems}

The evaluation of surveillance systems should promote the best use of public health resources by ensuring that only important problems are under surveillance and that surveillance systems operate efficiently (CDC, 2001). Most importantly, an evaluation should assess whether a system is serving a useful purpose while meeting the surveillance system's overall objectives. A surveillance system is useful if it contributes to the prevention and control of adverse health events, and if it increases awareness of the importance of controlling the occurrence of such an event (CDC, 2001). There are 
seven attributes identified by the CDC (2001) as important to consider when evaluating a surveillance system. The seven attributes which are necessary for an efficient and effective system are: adequate sensitivity, timeliness, representativeness, adequate positive predictive value, simplicity, acceptability, and flexibility.

According to the CDC (2001), sensitivity is the measurement of how many cases are picked up by the surveillance system, showing that any missed cases could alter the results of the surveillance system. The positive predictive value is the probability that an identified case truly is a case. If a surveillance system has high positive predictive values it will help enable health officials to focus on productive activities. To increase the usefulness of a surveillance system, it is important to ensure the system is accepted by those who use it, that it is simple to use and that it provides the health care professional with flexibility. A representative surveillance system will better characterize the epidemiological characteristics of an event in a certain population. A lack of representativeness could affect the usability of the information produced. Timeliness reflects the speed or delay between steps in a surveillance system. Timeliness is a component that may not be important in some programs, but may be critical in others.

Because surveillance systems vary widely in methodology, scope, and objectives, the criteria that are important to one system may be less important to another (CDC, 2001). Therefore, the success of a surveillance system may rely on an adequate balance of these attributes. As well, the strength of the evaluation depends on the ability 
of the evaluator to assess these attributes with respect to the system's objectives. It is important for the evaluator to understand that all seven attributes will not be appropriate for all surveillance systems (CDC, 2001).

The studies which were conducted and previously discussed on influenza immunization in the community did not report evaluating the surveillance systems used. This supports the need to increase research studies conducted in a community setting which evaluate an influenza immunization surveillance system. Without an evaluation component it is very difficult to determine if the surveillance system is serving a useful public health function while meeting the system's overall objectives.

\section{Summary}

Influenza has an impact on morbidity and mortality, hospitalization, health care costs and lost productivity and work time. Influenza vaccine is known to be effective, however, influenza vaccination rates are less than $90 \%$. Immunization promotional campaigns help increase influenza immunization rates, but health care providers must know who is or is not receiving vaccine in the community so they will know to which groups campaigns should be targeted.

To calculate the proportion of vaccine eligible groups that receive influenza vaccine, the number of individuals vaccinated (numerator) must be determined as well as the number of individuals eligible (denominator). Surveillance is a method of collecting numerator and denominator data. Some denominator data is readily available 
through existing population health data.

There were only a few influenza immunization surveillance methods in the community which have been reported in the literature. Buffington et al. (1991) found that when physicians used a poster to track the proportion of the target group vaccinated and sent out post card reminders to the target population it allowed them to track the proportion of the target group vaccinated. Physicians who used the poster and sent post card reminders also showed an increase in the amount of vaccine administered to the target population, compared to physicians in the control group who used computer generated billing codes at the end of the season to determine their target population. While this method was an ideal way to determine the proportion of target group vaccinated, it would not be practical because it would be very time consuming and it may be difficult to get the physicians' cooperation. Squires et al. (2001) used random digit dialling as a way to identify the proportion of a variety of target groups vaccinated. This method is not a practical way to collect influenza immunization data since it is very time consuming and expensive. MacDonald et al. (1996) used surveillance forms and were able to account for $72.7 \%$ of the vaccine distributed, but there was no feedback from participants regarding the forms or details regarding identification of target groups. Communicable Disease Control unit in Manitoba (2000) used a tally form to collect influenza immunization surveillance data, however, it was unclear what proportion of the doses were accounted for using the surveillance form. This method of surveillance would be a practical method to try again. However, it would be important to add a 
feedback component to the system and to evaluate the system to determine it's effectiveness in collecting influenza immunization data.

In conclusion, there are gaps in the literature regarding the best form of influenza immunization surveillance. In order to clarify who is receiving influenza vaccine and where to target influenza immunization campaigns, an influenza immunization surveillance system must be implemented and evaluated. The use of influenza immunization surveillance forms would be a practical way to collect surveillance data in comparison to the two other forms of surveillance which have been implemented in previous studies. The evaluation of the surveillance initiative will help determine if influenza immunization surveillance is an effective way to retrieve useful numerator data. 


\section{CHAPTER 3}

\section{Methodology}

This chapter presents the methods used to conduct this study. The initiative being evaluated, the instruments used, the data collection procedure, and data analysis are described. The ethical considerations are also discussed.

\section{Design of the Study and the Surveillance Initiative}

This descriptive, prospective study was designed to assess and compare the effectiveness of three influenza immunization surveillance forms in accounting for the influenza vaccine distributed to health care professionals practising in the community. Initially the study was designed to test two surveillance forms (tally form and individual form) but at the beginning of the 2001-2002 influenza season, the government of Newfoundland and Labrador developed and distributed a form (government form) to all health care professionals that received influenza vaccine. In the Eastern Region of Health and Community Services this form was sent to the health care professionals that refused to complete the individual or tally forms. Therefore, community health nurses and physicians in the eastern region of the province were provided with one of three influenza immunization surveillance forms, which were completed on each patient receiving influenza vaccine during the 2001-2002 season. The completed forms were returned to the Medical Officer of Health $(\mathrm{MOH})$ at the end of the influenza 
immunization season. Once the forms were returned, health care professionals were contacted to provide feedback on the surveillance forms.

\section{Setting and Respondents}

The study was conducted in the communities under the geographic boundaries of Health and Community Services Eastern, Newfoundland and Labrador. There are approximately 65 clinics/practices in this area of the province. These clinics consist of family physicians and community health nurses who administer influenza vaccine annually to individuals residing in the community. All practices were sent surveillance materials with their annual vaccine order. Those who returned completed surveillance forms were considered respondents in this study.

\section{Surveillance Forms}

There were three influenza immunization surveillance forms used. These were the tally form, found in appendix $\mathrm{B}$, individual form, found in appendix $\mathrm{C}$, and the government form, found in appendix D. The tally and the individual forms were designed for this study. These two surveillance forms contained the same information, however, each form had a different format. The tally form allowed the health care professional to document information on 12 patients per form, whereas health care professionals who completed the individual form completed one form per patient. Both forms contained the items of age, sex, reason for immunization, and date of 
immunization. Health care professionals also had an opportunity to indicate on the tally form and individual form if the patient received the influenza vaccine in the previous year, or had received pneumococcal vaccine in the past.

The third form was the government form which consisted of two columns, one to indicate if patients were over 65 years of age and the other to indicate if patients were under 65 years of age. The health care professional was required to add up the number of patients who received vaccine and write the total numbers in each column after every clinic. No patient specific identifying information was recorded on any of the three surveillance forms.

\section{Data Collection Procedure: Immunization Surveillance}

The first step in data collection consisted of sending health care professionals a letter from the $\mathrm{MOH}$, found in appendix E, explaining the influenza immunization surveillance initiative for the 2001-2002 season. This letter was sent with the influenza immunization order forms that are sent annually. Practices were stratified into two groups: one group consisted of practices with three or fewer health care professionals, and the other group consisted of practices with more than three health care professionals. By using random allocation, half of the practices in each stratum received the tally form

and the other half received the individual form. A sample copy of the surveillance form and the letter from the $\mathrm{MOH}$ was sent out to each health care provider with the letter explaining the study, found in appendix F. Within the first week, after all forms and 
information had been sent out, all health care professionals were contacted to determine if they had any questions about the study and if they were going to participate. If health care professionals stated or provided notification that they were not going to complete the tally or individual forms, they were sent the government form to complete. Health care professionals were not asked to provide an explanation as to why they refused to completed the forms used in this study.

After the study explanation was sent out, along with a copy of the surveillance form, health care professionals were sent copies of their surveillance forms with their vaccine order. Health care professionals were also sent a minimum of five appropriately addressed stamped envelopes to use to return completed forms.

Completed influenza immunization surveillance forms were returned to the MOH every month. Every practice received a monthly reminder by fax to complete surveillance forms for each dose of vaccine given and to return completed forms. A final fax was sent at the end of the influenza season to remind health care professionals to return completed surveys and to return unused vaccine.

As a final part of data collection, the communicable disease control nurses that work in the other Health and Community Services Boards in the province of Newfoundland and Labrador were contacted to determine how many government forms they had received from health care professionals, as well as how many doses of influenza vaccine they accounted for by using the government form. 


\section{Data Collection: Evaluation of Surveillance Forms}

The final stage of the data collection occurred at the end of the immunization season and when all potential surveillance forms were returned. Once the forms were returned, each health care professional was contacted to provide feedback. There was one questionnaire used for study respondents, found in appendix G, and a separate one for non-respondents, found in appendix $\mathrm{H}$. The respondents' questionnaire consisted of a number of open ended questions. These questions were developed as a way to determine if the form of surveillance used was an effective way to account for all the influenza vaccine distributed and if the respondents felt that they would be willing to use the same surveillance form in the future. This questionnaire also determined who completed the surveillance forms (office staff, physicians or nurses) and how the health care professionals felt about influenza immunization surveillance in general.

The questionnaire for the non-respondents consisted of two open ended questions. This questionnaire gave non-respondents the opportunity to provide suggestions on ways to facilitate their participation in the future. In addition, the questionnaire determined how these health care professionals felt about influenza immunization surveillance.

\section{Data Analysis}

The data was entered into a database, then coded and "cleaned" prior to analysis. The Statistical Package for Social Sciences (SPSS) was used for statistical analysis. The 
investigator performed all the data analysis. Descriptive statistics were used to summarize the percentage of influenza vaccine doses accounted for and administered and to summarize the characteristics of patients who received influenza vaccine. This data was compared for the three surveillance form formats. Fisher's Exact Test was used to assess the statistical significance of the difference in the proportion of doses accounted for and administered using each form and the odds ratio was used to assess the magnitude of any differences found. Content analysis was used to summarize the feedback received from the interview questionnaires.

\section{Ethical Considerations}

A written consent was not obtained in this study, however, elements of informed consent were adhered to. By using letters of explanation and follow up phone calls to clarify any concerns practices may have had about completing surveillance forms, health care professionals were informed of the study's association with a Masters in Nursing research study. The letters of explanation also clearly explained what the health care professionals' participation involved.

Health care professionals could refuse to participate by not returning completed forms. If health care professionals did not complete the individual or tally form they were sent the government form to complete. Health care professionals could also refuse to complete the government form. Consent was implied by the return of completed forms. Health care professionals could withdraw from the study at any time by not 
returning completed forms. Verbal consent was obtained from every health care professional before the telephone interview took place. Health care professionals received a fax consisting of the interview questions several days before the interview which provided them with an opportunity to consider their interest in providing feedback.

There was no penalty associated with not participating in this study and health care professionals did receive influenza vaccine as usual. Also, there was no penalty associated with not returning unused vaccine, since all unused vaccine is normally destroyed.

There was no harm associated with participating in this study. Influenza immunization surveillance forms only took a couple of minutes to complete and did not significantly add to the workload of healthcare professionals. There were several potential benefits for health care providers participating in this study. Respondents were provided with an opportunity to provide feedback on the immunization surveillance system used, and the initiative may have increased their awareness of vaccine eligible groups in their practices and, therefore, provided them with a chance to increase coverage rates. Patients may have benefited from the study as more vaccine eligible groups may have received vaccine if providers increased their coverage rates. Health and Community Services Eastern Region were provided with the opportunity to account for influenza vaccine doses distributed and the individuals receiving influenza vaccine. This data can assist health care professionals improve future vaccination rates in the community as they will know what groups should be targets for influenza immunization 
promotion campaigns.

Confidentiality was maintained in this study. The focus of this study was on practices and not individual health care professionals. There was no patient specific identifying information on the surveillance forms, nor was there any information identifying the health care professional completing the form. The surveillance forms were individually coded such that the investigator could assess the proportion of doses distributed to participating practices. A copy of the code identifiers were placed in a locked cabinet and used only for this aspect of data analysis. All the information received in this study was used for research purposes only. Data was reported in such a way that individual heath care providers and practices cannot be identified.

This study had the support of the Eastern Region of Health and Community Services who also provided financial support. A letter outlining financial support can be found in appendix I. Finally, this study proceeded with the approval of the Human Investigations Committee (HIC) of Memorial University of Newfoundland. The letter of approval from HIC can be found in appendix J. 


\section{CHAPTER 4}

\section{Results}

The study findings are presented in seven sections. The first section presents health care professionals' participation, which is followed by a section on the distribution and administration of the influenza vaccine. Next are sections on the recipients' profile, completeness of the data collected, participant feedback and feedback from other provincial health regions. Finally, there is a section on the proportion of target groups vaccinated.

\section{Participation}

In the 2001-2002 season, Health and Community Services Eastern Region distributed influenza vaccine to 51 physicians and 15 community health nurses practising in the community. The individual and tally forms were sent to all of these physicians and community health nurses. Overall, $91.2 \%$ of health care professionals participated and returned surveillance forms. The community health nurses who were sent surveillance forms were all employed by Health and Community Services Eastern Board: All of the community health nurses who were sent surveillance forms participated by returning their randomly assigned forms. Forty percent ( 6 of 15) of nurses received and returned the tally form, and 60\% (9 of 15) received and returned the individual form.

Of all the physicians who were sent the tally and individual forms, $62.7 \%$ (32 of 
51) returned their randomly assigned forms and $19.6 \%(10$ of 51$)$ returned the government form. All of these physicians were considered participants. Overall, $82.4 \%$ of physicians participated and returned surveillance forms. A total of $17.6 \%$ ( 9 of 51 ) of the physicians did not return any form and were considered non-participants.

Table 1 shows that the distribution of forms between participating physicians and all physicians who originally received forms were similar for all forms. For example, there were similar proportions of participating physicians, $40.4 \%$ (17 of 42 ), and all physicians, $45.1 \%$ (23 of 51), that had the tally form.

\section{Table 1}

Distribution of Forms Between Participating Physicians and All Physicians Who Were Originally Distributed Forms

\begin{tabular}{|c|c|c|c|c|c|}
\hline & & Tally & Individual & Government & Total \\
\hline \multirow{2}{*}{$\begin{array}{l}\text { Participating }{ }^{1} \\
\text { Physicians }\end{array}$} & $\mathrm{n}$ & 17 & 15 & 10 & 42 \\
\hline & $\%$ & 40.4 & 35.7 & 23.8 & 100 \\
\hline \multirow[t]{2}{*}{ All Physicians ${ }^{2}$} & $\mathrm{n}$ & 23 & 18 & 10 & 51 \\
\hline & $\%$ & 45.1 & 35.3 & 19.6 & 100 \\
\hline
\end{tabular}

${ }^{1} \mathrm{n}$ and $\%$ are the number and proportion of 42 physicians who participated and who received the specified form.

${ }^{2} \mathrm{n}$ and $\%$ are the number and proportion of all the 51 physicians who were sent surveillance forms and received the specified form.

Table 2 outlines the participation and non-participation of physicians in small and large clinics by the type of surveillance form they completed. There was no statistically significant difference between physicians practising in large and small clinics and 
whether or not they participated $(\mathrm{p}=0.41)$. Therefore, physicians practising in larger and smaller clinics were equally likely to participate.

\section{Table 2}

Participation and Non-participation of Physicians in Small and Large Clinics by Form Type

\begin{tabular}{|l|l|l|l|l|l|l|l||l|l|}
\hline & & \multicolumn{2}{|c|}{ Tally $^{3}$} & \multicolumn{2}{|c|}{ Individual $^{4}$} & \multicolumn{2}{|c|}{ Government $^{5}$} & \multicolumn{2}{|c|}{ Total } \\
\hline \multirow{4}{*}{$\begin{array}{l}\text { Participating } \\
\text { Physicians }\end{array}$} & $\begin{array}{l}\text { Small } \\
\text { Clinic }\end{array}$ & 7 & $\mathbf{4 1 . 2}$ & 11 & $\mathbf{7 3 . 3}$ & 6 & $\mathbf{6 0}$ & 24 & $\mathbf{5 7 . 1}$ \\
\cline { 2 - 11 } & $\begin{array}{l}\text { Large }^{2} \\
\text { Clinic }\end{array}$ & 10 & $\mathbf{5 8 . 8}$ & 4 & $\mathbf{2 6 . 7}$ & 4 & $\mathbf{4 0}$ & 18 & $\mathbf{4 2 . 8}$ \\
\hline $\begin{array}{l}\text { Non- } \\
\text { Participating } \\
\text { Physicians }\end{array}$ & $\begin{array}{l}\text { Smal1 } \\
\text { Clinic }\end{array}$ & 3 & $\mathbf{6 0 . 0}$ & 3 & $\mathbf{7 5 . 0}$ & 0 & 0 & 6 & $\mathbf{6 6 . 7}$ \\
\cline { 2 - 11 } & $\begin{array}{l}\text { Large } \\
\text { Clinic }\end{array}$ & 2 & $\mathbf{4 0 . 0}$ & 1 & $\mathbf{2 5 . 0}$ & 0 & 0 & 3 & 33.3 \\
\hline
\end{tabular}

${ }^{1}$ Clinics with 2 or less physicians practising.

${ }^{2}$ Clinics with 3 or more physicians practising.

${ }^{3} \mathrm{n}$ and $\%$ are number and proportion of 17 participating physicians or 5 non-participating physicians who had the tally form.

${ }^{4} \mathrm{n}$ and $\%$ are number and proportion of 15 participating physicians or 4 non-participating physicians who had the individual form.

${ }^{5} \mathrm{n}$ and $\%$ are number and proportion of 10 participating physicians who had the government form

\section{Distribution and Administration of Influenza Vaccine}

There were 13,310 doses of influenza vaccine distributed to physicians and community health nurses practising in the community during the 2001-2002 influenza season. Of these 13,310 doses, $77.2 \%(10,270$ of 13,310$)$ were distributed to physicians 
and community health nurses who returned the influenza immunization surveillance forms, while $22.8 \%$ ( 3,040 of 13,310$)$ were distributed to physicians who did not return their surveillance forms. Of the total 10,270 distributed to health care professionals who participated, there were 472 of 10,270 doses distributed to participating community health nurses and 9,798 of 10,270 doses distributed to participating physician's offices. The influenza vaccine distribution ranged from 1,130 doses to 10 doses which were sent to individual offices and the median number of doses distributed was 200. The largest amount of vaccine distributed to one office was 1,130 doses. This was distributed to an office where there were 2 physicians practising.

Of the total 13,310 doses distributed, $51.3 \%(6,824$ of 13,310$)$ were known to be administered. There were four physicians who returned unused vaccine. This returned vaccine when added to the vaccine they documented as administered, will be referred to as "administered + returned" throughout this chapter and the chapters that follow. There was $57.5 \%(7,645$ of 13,310$)$ of vaccine administered + returned. If one considers only the 10,270 doses distributed to participating health care professionals, $66.4 \%$ (6824 of 10,270 ) of the doses were known to be administered by using the three surveillance forms and $74.3 \%$ ( 7634 of 10,270$)$ were administered + returned. Of those distributed to community health nurses, $95.5 \%$ ( 451 of 472$)$ had been administered, whereas participating physicians administered $65.0 \%$ (6373 of 9798 ) of the doses they had been distributed. The remainder of this section summarizes the results with the denominator of 10,270 doses which were distributed to health care professionals who participated. 
Table 3 summarizes the distribution of influenza vaccine, as well as the doses administered and administered + returned, to health care professionals using the three surveillance forms. The highest proportion of influenza vaccine, $40 \%(4,150$ of 10,270$)$, was distributed to those health care professionals who received the individual form.

\section{Table 3}

Influenza Vaccine Distributed, Administered and Administered+Returned by Health Care Professionals Using the Three Surveillance Forms

\begin{tabular}{|l|l|l|l|l||l|}
\hline & & Tally & Individual & Government & Total \\
\hline \multirow{2}{*}{ Doses Distributed } & $\mathrm{n}^{1}$ & 3,650 & 4,150 & 2,470 & 10,270 \\
\cline { 2 - 6 } & $\%^{1}$ & $\mathbf{3 5 . 5}$ & $\mathbf{4 0 . 4}$ & $\mathbf{2 4 . 1}$ & $\mathbf{1 0 0}$ \\
\hline \multirow{3}{*}{ Doses Administered } & $\mathrm{n}^{2}$ & 2,292 & 2,409 & 2,123 & 6,824 \\
\cline { 2 - 6 } & $\%^{2}$ & $\mathbf{6 2 . 7}$ & $\mathbf{5 8 . 1}$ & $\mathbf{8 6 . 0}$ & $\mathbf{6 6 . 4}$ \\
\hline \multirow{2}{*}{$\begin{array}{l}\text { Doses } \text { Administered } \\
\text { +Returned }\end{array}$} & $\mathrm{n}^{3}$ & 2,352 & 3,159 & 2,123 & 7,634 \\
\cline { 2 - 6 } & $\%^{3}$ & $\mathbf{6 4 . 4}$ & $\mathbf{7 6 . 1}$ & $\mathbf{8 6 . 0}$ & $\mathbf{7 4 . 3}$ \\
\hline
\end{tabular}

${ }^{1} \mathrm{n}$ and $\%$ are the number and proportion of 10,270 doses distributed to health care professionals, by form type.

${ }^{2} n$ and $\%$ are the number and proportion of doses distributed (tally $n=3650$, individual $n=4150$, government $n=2470$ ) that were administered by health care professionals, by form type.

${ }^{3} \mathrm{n}$ and $\%$ are the number and proportion of doses distributed (tally $\mathrm{n}=3650$, individual $\mathrm{n}=4150$, government $n=2470$ ) that were administered + returned by health care professionals, by form type.

Table 3 shows that of the 10,270 doses of vaccine distributed to participating health care professionals, $66.4 \%(6,824$ of 10,270$)$ were documented as administered on returned surveillance forms. Health care professionals that completed the tally form 
documented that they administered $62.7 \%(2,292$ of 3,650$)$ of the doses distributed to them. Health care professionals who completed the individual form documented that they administered $58.1 \%(2,409$ of 4,150$)$ of the doses distributed to them. The highest proportion of distributed doses of influenza vaccine, $86.0 \%(2,123$ of 2,470$)$, was documented by the health care professionals who completed the government form, all of whom were physicians.

Table 3 shows that the government form identified a much higher proportion of doses administered $(86.0 \%)$ than the tally $(62.7 \%)$ or the individual forms $(58.1 \%)$. The difference between the proportion of doses documented by health care professionals who completed the tally form and the individual form compared to the government form was statistically significant $(\mathrm{p}<0.0005)$. In addition, the difference in the proportion of doses documented by health care professionals who completed the individual form compared to the tally form was also statistically significant $(\mathrm{p}<0.0005)$.

The vaccine administered + returned consisted of returned vaccine and administered vaccine. There were a total of $830(6.2 \%)$ doses returned from the 13,310 doses distributed; twenty doses of the 830 returned were from non-participating physicians. Of the 810 doses returned from physicians who participated, 750 doses $(92.6 \%)$ were returned from two physicians who had completed the individual form and $60(7.4 \%)$ were returned from one physician who completed the tally form. There were 810 of $10,270(8.1 \%)$ doses of influenza vaccine returned from participating health care professionals, with 710 of these doses returned by one physician's clinic. The remaining 
100 doses came from two separate physicians' clinics. A larger quantity of vaccine was returned by physicians who completed the individual form, with $76.1 \%$ of the distributed doses administered + returned, compared to $58.1 \%$ documented as administered by health care professionals who completed the individual form, as shown in Table 3. With only a few doses of vaccine returned by those who completed the tally form, $64.4 \%$ were administered + returned compared to the $62.7 \%$ that were administered. Overall, the health care professionals who completed the government form administered + returned $86.0 \%(2,123$ of 2,470$)$ of distributed doses compared to $64.4 \%(2,352$ of 3,650$)$ which were administered and returned by health care professionals that completed the tally form. The difference between the proportion of doses administered + returned by health care professionals who completed the government form (86.0\%) and those who completed the individual (76.1\%) and tally forms (64.4\%) was statistically significant $(\mathrm{p}<0.0005)$.

Figure 1 illustrates graphically the information presented in Table 3. It can been seen that health care professionals who completed the government form accounted for and administered the greatest proportion of the vaccine which they had been distributed compared to those who received the tally and individual forms. 


\section{Figure 1}

Proportion Of Distributed Influenza Vaccine Doses That Were Administered, And Administered + Returned Using The Three Surveillance Forms

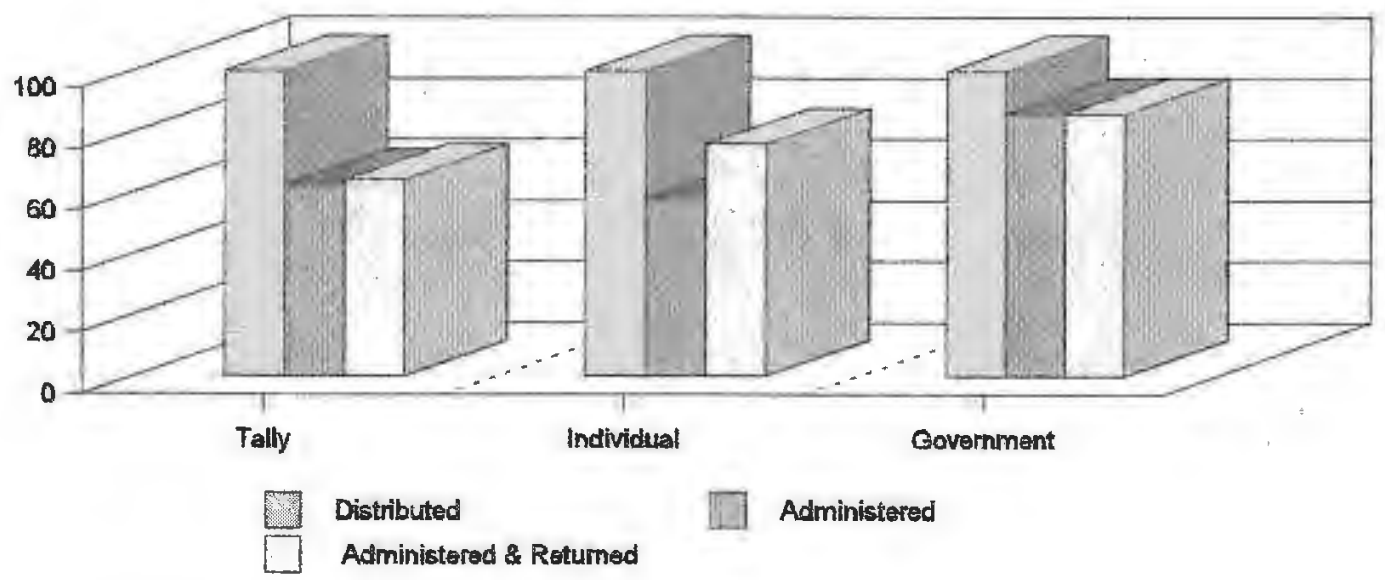

\section{Recipient Profile}

Reasons why individuals received the influenza vaccine were identified on the indivichal and tally forms. The government form did not have a section for health care professionals to provide the reason why individuals received the vaccine. Health care professionals that completed the government form were only required to identify whether or not the individual receiving the vaccine was over or under 65 years of age.

Age was thus the only item identified by all three surveillance forms. Of the 
6,824 doses of influenza vaccine known to have been administered in the 2001-2002 influenza season, 6,719 (98.4\%) individuals had information provided regarding age. Of these 6,719 individuals, $2,716(40.4 \%)$ who received the influenza vaccine were under 65 years of age and $4,020(59.8 \%)$ were 65 years of age and older. The government form identified 1,379 of $4,020(34.3 \%)$ individuals who were 65 years of age and older and 744 of $2,716(27.4 \%)$ of those under 65 years of age that received influenza vaccine. The tally form identified 1,262 of $4,020(31.4 \%)$ that were over 65 years of age and 955 of $2,716(35.2 \%)$ that were under 65 years of age. The individual form identified 1,379 of $4,020(34.3 \%)$ that were 65 years of age and older and 1,017 of $2,716(37.4 \%)$ that were under 65 years of age.

Health care professionals who completed the individual and tally forms had a space provided so they could identify the recipient's date of birth as well as providing the reason for receiving the vaccine. Age was one of the reasons provided for individuals receiving influenza vaccine. There were 4,701 doses of influenza vaccine administered by health care professionals who completed the individual and tally forms. The mean age for individuals receiving influenza vaccine was 64.8 years of age. The ages ranged from 3 to 102 years.

Table 4 outlines the reasons why health care professionals who completed individual and tally forms administered influenza vaccine. 
Table 4

Reasons Individuals Received Influenza Vaccine Indicated by Health Care Professionals who Completed Tally and Individual Forms

\begin{tabular}{|c|c|c|c|c|c|c|}
\hline \multirow[b]{2}{*}{ Reason } & \multicolumn{2}{|c|}{ Tally Form ${ }^{1,4}$} & \multicolumn{2}{|c|}{ Individual Form ${ }^{2,4}$} & \multicolumn{2}{|l|}{ Total } \\
\hline & $\mathrm{n}$ & $\%$ & $\mathrm{n}$ & $\%$ & $\mathrm{n}$ & $\%$ \\
\hline Age & 949 & $41.0 \%$ & 1001 & $41.5 \%$ & 1950 & $82.5 \%$ \\
\hline Cardiac & 649 & $28.3 \%$ & 517 & $21.5 \%$ & 1166 & $49.8 \%$ \\
\hline Pulmonary & 512 & $22.4 \%$ & 545 & $22.6 \%$ & 1057 & $45 \%$ \\
\hline Diabetes & 356 & $15.5 \%$ & 320 & $13.3 \%$ & 676 & $28.8 \%$ \\
\hline Other $^{3}$ & 314 & $13.7 \%$ & 315 & $12.8 \%$ & 629 & $26.5 \%$ \\
\hline $\begin{array}{l}\text { Long Term Care } \\
\text { Facility }\end{array}$ & 131 & $5.7 \%$ & 162 & $6.7 \%$ & 293 & $12.4 \%$ \\
\hline $\begin{array}{l}\text { Health Care } \\
\text { Professional }\end{array}$ & 123 & $5.4 \%$ & 115 & $4.8 \%$ & 238 & $10.2 \%$ \\
\hline Cancer & 118 & $5.2 \%$ & 105 & $4.4 \%$ & 223 & $9.6 \%$ \\
\hline Renal & 70 & $3.1 \%$ & 59 & $2.4 \%$ & 129 & $5.5 \%$ \\
\hline
\end{tabular}

$\mathrm{n}$ and $\%$ is the number and proportion of 2292 patients with reasons identified by health care professionals who completed the tally form.

${ }^{2} \mathrm{n}$ and $\%$ is the number and proportion of 2409 patients with reasons identified by health care professionals who completed the individual form.

${ }^{3}$ Other is any reason health care professionals had for administering vaccine to their patient that was not identified as a reason on the form. Health care professionals were provided with a space where they could write in their other reason.

${ }^{4}$ The total $\%$ does not add up to $100 \%$ because many patients had more than one reason identified by health care professionals who completed both forms. 
Of the individuals with age identified as the reason for receiving influenza vaccine, $64.8 \%(1,263$ of 1,950$)$ had other reasons identified as well. Therefore only $35.2 \%(687$ of 1,950$)$ of individuals had age identified as the only reason for receiving influenza vaccine. Similar proportions of health care professionals who completed the tally form, $41.0 \%$ (949 of 2,292), and the individual form, $41.5 \%(1,001$ of 2,409$)$ identified age as the main reason why they administered influenza vaccine to their patients. Four percent $(78$ of 1,950$)$ of health care professionals identified age as the only reason why their patient received vaccine despite the fact that the individual receiving the vaccine was under 65 years of age.

Table 4 shows that chronic diseases such as cardiac disorders, pulmonary disorders and diabetes showed slight differences in proportions by form type. Other chronic conditions such as cancer and renal disease accounted for a total of $9.6 \%$ and $5.5 \%$ of the reasons provided. The category "other" accounted for a total of $26.5 \%$ of reasons and $4.3 \%$ of these reasons were specified. Examples of the "other" reasons for receiving the vaccine consisted of chronic conditions such as lupus, anemia, multiple sclerosis, as well as individuals who were in close contact with those who were susceptible to contracting the influenza virus. Health care professionals only accounted for a total of $10.2 \%$ of the reasons identified.

Overall, 3,416 of $4,701(72.7 \%)$ forms returned provided reasons as to why their patient received the influenza vaccine. Of these 3,416 there were 1,252 (26\%) patients who had more than one reason for receiving the influenza vaccine. More specifically, 
$905(19.3 \%)$ had 2 identified reasons, $283(6 \%)$ had 3 identified reasons, $51(1.1 \%)$ had 4 identified reasons, and $13(0.27 \%)$ had 5 identified reasons.

In addition to the reasons why they were given vaccine, the patient's sex was identified by those health care professionals who completed the individual and tally forms. Of the 4,617 individuals who had their sex identified, 2,577 (55.8\%) were female and $2,040(44.2 \%)$ were male.

The individual and tally forms also provided health care professionals with an opportunity to identify whether or not their patients received the influenza vaccine in the past season and if they had ever received pneumococcal vaccine. There were health care professionals who did not complete some of the questions on the surveillance forms, therefore, the denominator may change depending on the amount of missing data. Health care professionals who completed the tally or individual forms indicated that $84.6 \%$ $(3,928$ of the 4,643$)$ of the individuals who received influenza vaccine in 2001-2002 also received the vaccine in the previous year. Only $15.4 \%(713$ of 4,641$)$ of those who received the vaccine in 2001-2002 did not receive it in the previous year.

The majority, $70.9 \%(3,111$ of 4,383$)$, of individuals who received influenza vaccine in 2001-2002 did not receive pneumococcal vaccine in the past. There were 1,277 of $4,388(29.1 \%)$ of those individuals who received influenza vaccine in the 2001 2002 season that had received pneumococcal vaccine in the past. Of those 1,277 who received pneumococcal vaccine in the past, $999(78.2 \%)$ were over 65 years of age in 2001-2002. There were, however, $47.7 \%(1,465$ of 3,093) of individuals over 65 years of 
age who did not receive pneumococcal vaccine in the past.

\section{Completeness of Data}

There were data missing from some of the individual and tally forms that were returned. Unlike the individual and tally forms, the government form only consisted of two columns, one for individuals 65 years and over and one for individuals 65 years and younger. When completing the government form, health care professionals were required to tally up the doses they administered after each clinic and fill in the number on the form. Therefore, there were no missing data due to the nature of the form. The individual and tally forms had six questions which the health care professional had to complete. The completed forms that were returned did have missing data from each item. There were 54 forms returned that had more than one item missing. Table 5 outlines the proportion of data missing from each item on the individual and tally forms. There was a higher proportion of missing data from the health care professionals who completed the tally form, since 346 of 2,292 (15.0\%) tally forms had at least one piece of data missing, compared to those who completed the individual forms, where 256 of $2,409(10.6 \%)$ forms had at least one piece of data missing. An important question was the one related to reason for receiving the vaccine. There was very little missing data on the tally form $(0.4 \%)$ or individual form (1.0\%) related to this item. The majority of data was missing from the item related to whether the patient had received pneumococcal vaccine, with 170 of $2,292(7.4 \%)$ entries missing from the tally forms 
and 148 of $2,409(6.1 \%)$ missing from the individual forms.

\section{Table 5}

Proportion of Missing Data For Each Question by Tally and Individual Forms

\begin{tabular}{|l|l|l|l|l|}
\hline \multirow{2}{*}{} & \multicolumn{2}{|l|}{ Tally } & \multicolumn{2}{l|}{ Individual $^{2}$} \\
\cline { 2 - 5 } & $\mathrm{n}$ & $\%$ & $\mathrm{n}$ & $\%$ \\
\hline Age & 91 & $\mathbf{3 . 9}$ & 14 & $\mathbf{0 . 6}$ \\
\hline Date of Vaccine & 0 & $\mathbf{0}$ & 4 & $\mathbf{0 . 2}$ \\
\hline Reason & 9 & $\mathbf{0 . 4}$ & 24 & $\mathbf{1 . 0}$ \\
\hline Sex & 42 & $\mathbf{1 . 8}$ & 42 & $\mathbf{1 . 7}$ \\
\hline Pneumococcal Vaccine & 170 & $\mathbf{7 . 4}$ & 148 & $\mathbf{6 . 1}$ \\
\hline Influenza Vaccine & 34 & $\mathbf{1 . 5}$ & 24 & $\mathbf{1 . 0}$ \\
\hline Total & 346 & $\mathbf{1 5 . 0}$ & 256 & $\mathbf{1 0 . 6}$ \\
\hline
\end{tabular}

' $\mathrm{n}$ and $\%$ are number and proportion of forms with the identified information missing from the 2292 tally forms completed.

${ }^{2} \mathrm{n}$ and $\%$ are number and proportion of forms with the identified information missing from the 2409 individual forms completed.

Table 6 outlines the proportion of missing data from each question on forms completed by physicians and community health nurses. Both physicians, $13.1 \%$ (558 of 4250 ), and community health nurses, $9.8 \%$ (44 of 451), had similar proportions of forms returned with missing data. 


\section{Table 6}

Proportion of Missing Data For Each Question, by Physician and Community Health Nurse

\begin{tabular}{|l|l|l|l|l|}
\hline \multirow{2}{*}{} & \multicolumn{2}{|l|}{ Physicians $^{1}$} & \multicolumn{2}{l|}{$\begin{array}{l}\text { Community } \\
\text { Health Nurses }\end{array}$} \\
\cline { 2 - 5 } & $\mathrm{n}$ & $\%$ & $\mathrm{n}$ & $\%$ \\
\hline Age & 104 & $\mathbf{2 . 4}$ & 1 & $\mathbf{0 . 2}$ \\
\hline Date of Vaccine & 4 & $\mathbf{0 . 1}$ & 0 & $\mathbf{0}$ \\
\hline Reason & 27 & $\mathbf{0 . 6}$ & 6 & 1.3 \\
\hline Sex & 76 & $\mathbf{1 . 8}$ & 8 & 1.7 \\
\hline Pneumococcal Vaccine & 296 & $\mathbf{7 . 0}$ & 22 & $\mathbf{4 . 9}$ \\
\hline Influenza Vaccine & 51 & $\mathbf{1 . 2}$ & 7 & 1.6 \\
\hline Total & 558 & $\mathbf{1 3 . 1}$ & 44 & $\mathbf{9 . 8}$ \\
\hline
\end{tabular}

' $\mathrm{n}$ and $\%$ are number and proportion of forms with identified information missing from the 4250 forms completed by physicians.

${ }^{2} \mathrm{n}$ and $\%$ are number and proportion of forms with identified information missing from the 451 forms completed by community health nurses.

Table 7 outlines the proportion of missing data from each question, by clinic size. Both large, $14.5 \%$ (176 of 1218), and small clinics, $12.2 \%$ (426 of 3483 ) had similar proportions of forms returned with missing data. 
Table 7

Proportion of Missing Data From Each Question, by Clinic Size

\begin{tabular}{|l|l|l|l|l|}
\hline \multirow{2}{*}{} & \multicolumn{2}{|l|}{ Small Clinics $^{1}$} & \multicolumn{2}{l|}{ Large Clinics } \\
\cline { 2 - 5 } & $\mathrm{n}$ & $\%$ & $\mathrm{n}$ & $\%$ \\
\hline Age & 75 & $\mathbf{2 . 2}$ & 30 & $\mathbf{2 . 5}$ \\
\hline Date of Vaccine & 4 & $\mathbf{0 . 1}$ & 0 & $\mathbf{0}$ \\
\hline Reason & 29 & $\mathbf{0 . 8}$ & 4 & $\mathbf{3 . 3}$ \\
\hline Sex & 59 & $\mathbf{1 . 7}$ & 25 & $\mathbf{2 . 1}$ \\
\hline Pneumococcal Vaccine & 219 & $\mathbf{6 . 3}$ & 99 & $\mathbf{8 . 1}$ \\
\hline Influenza & 40 & $\mathbf{1 . 2}$ & 18 & $\mathbf{1 . 5}$ \\
\hline Total & 426 & $\mathbf{1 2 . 2}$ & 176 & $\mathbf{1 4 . 5}$ \\
\hline
\end{tabular}

${ }^{1} \mathrm{n}$ and $\%$ are number and proportion of forms with the identified information missing from the 3483 forms that were completed by health care professionals in small clinics.

${ }^{2} \mathrm{n}$ and $\%$ are number and proportion of forms with the identified information missing from the 1218 forms that were completed by health care professionals in large clinics.

\section{Participant Feedback}

All of the health care professionals that were sent influenza immunization surveillance forms were contacted to provide feedback on the forms. A total of 46 of the 66 health care professionals (69.6\%) provided feedback. All of the 15 community health nurses that were sent surveillance forms provided feedback, and of the 51 physicians that were sent surveillance forms, $31(60.7 \%)$ responded to the feedback questionnaire. The 20 physicians that did not provide feedback were contacted, however, they did not return 
phone calls or return the copies of the questionnaires they had been faxed.

Of the 51 physicians who were sent surveillance forms, $9(17.6 \%)$ did not complete and return the tally or individual surveillance forms. Of those 9, only 2 (22.2\%) physicians provided feedback. There were 29 of the $42(69.0 \%)$ physicians who completed surveillance forms that provided feedback on the surveillance forms.

All of the community health nurses responded to the feedback questionnaire through a telephone interview, whereas, 26 of the $31(83.8 \%)$ physicians that responded to the questionnaire were interviewed by telephone and the other 5 physicians (16.1\%) were faxed a copy of the interview questions and returned them via fax because they were unable to be reached by telephone.

The feedback which was received from health care professionals looked at the accuracy of data collected by using the surveillance forms and the acceptability of surveillance. In determining the accuracy of the data, all of the community health nurses provided feedback on the surveillance forms and they all felt that they were able to account for the vaccine they administered by using the forms. The majority of physicians who provided feedback, $79.3 \%$ ( 23 of 29 ), felt they were able to account for most of the vaccine they administered by using the surveillance forms. There were $20.7 \%$ ( 6 of 29 ) of physicians that stated they were not able to account for the vaccine they administered by using the surveillance forms they had been provided. One physician forgot to complete the tally forms he had received for the first few clinics and stated he did keep a list of those he had vaccinated so he completed the forms from the list. The other 
physicians stated reasons such as they forgot to complete forms on several occasions, or they forgot to carry forms to their home visits and personal care homes for completion. In addition, one physician who completed the government form indicated that he administered more vaccine ( 20 doses) than he actually received.

The acceptability of surveillance was also determined through the feedback provided by health care professionals. The majority of community health nurses, $86.6 \%$ (13 of 15), and physicians, $72.4 \%$ ( 21 of 29 ), that provided feedback revealed they would like to see the same surveillance form used in the future. The two nurses who did not feel they would like to see the form used again both completed the individual form and felt that the form would be easier to complete if it was formatted as a tally sheet. They stated that there was too much paper and there would be less paper if information for several individuals was condensed on one sheet. The eight physicians who felt they would not like to use the form in the future consisted of six physicians who completed the individual forms and two who completed the tally forms. They stated several reasons why they would like to see the forms changed. They felt that fewer items to complete on each patient would be better, and those who completed the individual form would have liked a tally form type or would like a form that would not take so much time to complete.

All the community health nurses felt that influenza immunization surveillance in general is a good idea. Two of the nurses elaborated on their opinion of influenza immunization surveillance by stating that it is important to determine the number of 
doses of influenza vaccine administered and to whom they are administered as it should help prepare for future pandemics.

The majority of physicians, 22 of the $29(75.8 \%)$ who completed the forms, felt that influenza immunization surveillance was a good idea. They felt that surveillance was a good way to collect statistical information about influenza vaccinations, as well as a good way to determine who is not getting vaccinated if there is a way to determine denominator data. Two physicians stated that surveillance was a great idea and they had been collecting this information for their own practices for the past few years as a way to keep track of who was receiving the vaccine.

Seven of the $29(24.1 \%)$ participating physicians felt that they could not see the benefit of influenza immunization surveillance. They felt that surveillance was not important and they could not see the benefit of such a surveillance system. They also felt that they did not have time to collect influenza immunization surveillance data, and that doctors do not appreciate another task or form to complete.

There were two physicians who answered the questions on the non-respondent questionnaire. One of the physicians stated that he did not have a problem with the individual form which he had been sent, however he did not receive the forms until after his influenza vaccination clinic was completed. He was, therefore, unable to complete the forms and stated he would participate in the future if he was to receive the forms on time. The other physician misunderstood how he was supposed to complete the tally forms he had been sent and only completed one of the tally sheets he received. He felt 
that he was only supposed to provide a cross section of the influenza vaccines he administered for the season rather than complete information on every patient vaccinated. Both physicians felt that influenza immunization surveillance is a good idea. However, one of the physicians stated he did not see influenza immunization surveillance as a priority.

The amount of time required to collect surveillance data was also an important factor when determining health care professionals' acceptance of surveillance. Participating community health nurses and physicians that provided feedback stated that it took approximately one minute to complete the information on each patient who received the influenza vaccine. The highest number of surveillance forms returned from one health care professional was 337 . These forms were completed by a physician. It therefore took that particular physician a total of 5.6 hours to complete the influenza immunization surveillance forms, spread over several weeks. This physician provided his influenza vaccinations from October 26, 2001 to December 6, 2001. The first week this physician vaccinated 155 patients which would amount to 2.5 hours that week spent on completing surveillance forms. The second week the physician vaccinated 165 patients which took him 2.75 hours that week to collect influenza immunization surveillance data. Therefore, the maximum amount of time spent on completing the surveillance forms was only 2.75 hours a week for two weeks of the entire year. The minimum amount of time spent on completing surveillance forms was ten minutes because the least number of forms sent in by a health care professional was ten. The 
average number of forms returned was 20.9 . Therefore, it only took health care professionals an average of 20.9 minutes to complete surveillance forms per influenza season.

There were 25 of the $29(86.2 \%)$ physicians who stated they completed the forms themselves, 3 of the $29(13.8 \%)$ stated that their secretary was responsible for completing the forms, and $1(3.4 \%)$ physician who stated a nurse was responsible for completing the forms. The physician who returned the most forms (337) did complete the forms himself, and all the community health nurses completed their forms.

In addition to the time taken by health care professionals who completed the forms, there was also time spent by those individuals of Health and Community Services Eastern Region, who were responsible for organizing, sending out and collecting the influenza immunization surveillance forms. It took approximately 12 hours to put together the information and forms and send it to the appropriate health care professionals. There was time involved in entering the surveillance data once the forms were returned. It took approximately one minute to enter the information on one form. There were 4701 forms returned that required data entry. Therefore, it took approximately 78.4 hours to enter the surveillance data, using 7.5 hour workdays this would equate to 10.5 work days. It took a total of three work days to get feedback from healthcare professionals and send out reminders to return forms. It therefore took a total of 112.9 hours, which amounts to 15 ( 7.5 hour) work days to carry out this entire surveillance initiative. 


\section{Feedback from Other Provincial Health Regions}

There were five other provincial Health and Community Services Regions that used the government influenza immunization surveillance forms in the 2001-2002 season. Communicable disease control nurses were contacted by phone for each of the five regions to obtain information regarding the proportion of forms returned and the doses of influenza vaccine accounted for by using the government form. Information was obtained for three of the five regions, however, the communicable disease control nurses contacted did not have precise data available to share. One of the regions only received approximately $30 \%$ of the forms they distributed to the health care professionals in their

region and there was only approximately $30 \%$ of the distributed vaccine accounted for by using the government form. The majority of the health care professionals in this region that administered influenza vaccine were physicians (personal communication, communicable disease control nurse, March 20, 2003).

One of the other regions that provided information about the surveillance forms showed that $40 \%$ of health care professionals returned influenza immunization surveillance forms with approximately $25 \%$ to $30 \%$ of the vaccine accounted for. The majority of health care professionals who administered influenza vaccine in this region were also physicians (personal communication, communicable disease control nurse, March 19, 2003).

The final health care board obtained $90 \%$ of the forms they distributed to health care professionals and accounted for nearly $90 \%$ of the vaccine that had been distributed 
(personal communication, communicable disease control nurse, March 19, 2003). This region was different than the two previous regions in that $84.2 \%$ of the health care professionals that administered vaccine in this region were community health nurses .

\section{Target Groups Vaccinated}

Although the purpose of this study was to assess the effectiveness of using surveillance forms to account for the influenza vaccine distributed, the results can be used to estimate vaccine coverage rates, since it provides numerator data on individuals 65 years of age and older and individuals living with diabetes.

Denominator data was available for age and individuals living with diabetes for the Eastern Region of Newfoundland and Labrador. There was no denominator data available for the other chronic diseases or target groups that was region specific. There were 16,905 individuals 65 years of age and older living in the Eastern region of the province of Newfoundland and Labrador in 1996 (Centre for Health Information, 2000). There were 4,020 individuals 65 years of age and older identified as receiving influenza vaccine in the community setting during the 2001-2002 season. Assuming that the number of individuals age 65 years and older residing in this region of the province has not changed significantly, only $25.0 \%$ (4220 of 16,905) of individuals in this target group were documented as receiving influenza vaccine for the 2001-2002 season.

There were 676 individuals with diabetes identified as the reason why they received influenza vaccine. In 2001, there were 6,269 individuals over the age of 12 
living with diabetes in the Eastern Region of the province (Canadian Institute for Health Information, 2001). Assuming that the denominator data was accurate, $10.7 \%$ of those with diabetes were identified as receiving the influenza vaccine because they had diabetes.

\section{Summary}

The results of this study showed an overall participation rate of $91.2 \%$, with a $100 \%$ response rate from the community health nurses and $82.4 \%$ from physicians. The non-participants did not significantly differ based on the forms they had been sent or the size of their clinic.

There were 13,310 doses of influenza vaccine distributed to health care professionals practising in the community during the $2001-2002$ season. Of these 13,310 doses, $51.3 \%$ were administered and $57.5 \%$ were administered + returned. There were 10,270 doses distributed to health care professionals who participated in the surveillance initiative, of which $66.4 \%$ were administered and $74.3 \%$ were administered + returned. Community health nurses administered $95.5 \%$ of the doses they had been distributed, whereas, physicians administered $65.0 \%$ of those they had been distributed.

Health care professionals who completed the government form documented the highest proportion, $86.0 \%$, of the vaccine distributed compared to $62.7 \%$ for the tally form and $58.1 \%$ for the individual form. The difference was statistically significant when comparing proportions documented for all three forms $(p<0.0005)$. 
There were a total of 810 doses returned by health care providers who participated. Those who completed the government form administered + returned a statistically significantly higher proportion, $86 \%$, of the vaccine they had been distributed, than those who completed the individual form, $76.1 \%$, and the tally form, $64.4 \%(\mathrm{p}<0.0005)$.

The individual and tally forms contained more items than the government form and thus provided more information about vaccine recipients. Age was the only item identified by all three surveillance forms. Over half the individuals, $59.8 \%$, who received influenza vaccine were over 65 years of age. Of the individuals with age identified as a reason for receiving influenza vaccine, $64.8 \%$ had other reasons identified as well. Health care professionals who completed the tally and individual forms indicated that $84.6 \%$ of those who received the vaccine this year also received the vaccine last year. In addition, $70.9 \%$ of individuals who received influenza vaccine in the 2001-2002 season did not receive pnemococcal vaccine in the past.

There were very little missing data from the individual and tally forms, especially for the question relating to the reason for immunization. There was no difference in the amount or type of data missing from the forms completed by community health nurses or physicians, nor was there a difference in the data missing from forms returned by small or large clinics.

All of the community health nurses who provided feedback felt they were able to account for the influenza vaccine they had administered by using the surveillance forms. 
Over half $(60.7 \%)$ of physicians provided feedback. Of the physicians who provided feedback, $79.3 \%$ felt that they could account for the vaccine they had administered by using the surveillance forms. Those who felt they could not account for the vaccine stated it was because they forgot to complete the forms on different occasions. The majority of community health nurses, $86.6 \%$, and $72.4 \%$ of the physicians that provided feedback revealed that they would like to see the same form used again. All of the community health nurses and $75.8 \%$ of the physicians interviewed felt that influenza immunization surveillance is a good idea.

The time taken to complete surveillance forms varied depending on the doses of vaccine administered by the health care professional. Health care professionals felt that the forms took one minute to complete and the most time spent on completing surveillance forms was 2.75 hours a week for two weeks of the entire year. There were approximately 15 ( 7.5 hour) workdays required to organize, send out, collect and enter surveillance data. This time also included reminding health care professionals to send out forms and collecting feedback on the surveillance initiative.

There were three of the five other regions in the province that provided information about their influenza immunization surveillance initiatives. This study accounted for considerably more vaccine than two of these provincial regions. There was one region that accounted for $90 \%$ of the vaccine distributed. This particular region had a higher proportion of community health nurses, $84.2 \%$, administering influenza vaccine than the Eastern region and the other two regions that provided feedback. 
Denominator data were available for individuals under and over 65 years of age and diabetics. Twenty five percent of individuals 65 years of age and over and $10.7 \%$ of diabetics who were residing in the Eastern region of the province were documented as receiving the influenza vaccine. 


\section{CHAPTER 5}

\section{Discussion}

This chapter presents a discussion of the study's findings. The first section will discuss the study's results in relation to the CDC (2001) guidelines for evaluating a surveillance system. The second section draws conclusions on how to improve influenza immunization surveillance. Finally, the third section discusses how to improve the surveillance of target groups.

\section{Evaluation of Surveillance Forms and Systems}

The purpose of this study was to assess a new surveillance system as a way to monitor who is receiving influenza vaccine. For such a system to be effective it must collect useful information, obtain complete and accurate data, and obtain data from everyone who administers influenza vaccine. To determine if the surveillance system used in this study was an effective system to collect influenza immunization surveillance data, it is important to evaluate the system. Therefore, the study's findings will be discussed in the context of CDC (2001) guidelines for evaluating a surveillance system. These guidelines include seven criteria that are considered important to follow when evaluating a surveillance system: sensitivity, adequate positive predictive value, simplicity, acceptability, flexibility, representativeness, and timeliness. Because all surveillance systems vary widely in methodology, scope, and objectives, criteria that are 
important to one system may be less important to another (CDC, 2001).

Sensitivity was one of the criteria used to evaluate the surveillance system and the influenza immunization surveillance forms used in this study. The sensitivity of a surveillance system can be defined as the system's ability to detect the proportion of cases of a disease or health condition, as well as the system's ability to detect epidemics (CDC, 2001). The surveillance system in this study was not designed to detect health conditions or epidemics, instead it was developed to account for the doses of influenza vaccine administered in the 2001-2002 season. Therefore, the sensitivity of the influenza immunization surveillance forms and the overall surveillance system used in this study were determined by evaluating its ability to account for the proportion of influenza vaccine doses administered and those administered + returned.

Positive predictive value refers to determining if an identified case is actually a true case (CDC, 2001). This criterion was applied to influenza vaccination surveillance, in terms of knowing if vaccine recipients truly received the vaccine.

The simplicity of a surveillance system refers to both its structure and ease of operation. The surveillance system should be as simple as possible while still meeting the system's overall objectives (CDC, 2001). The acceptability of the system reflects the willingness of the health care professionals in this study to participate and use the surveillance forms. The simplicity of the influenza immunization surveillance system used in this study will be discussed along with the system's acceptability to health care professionals. 
Flexibility is another criterion that was used to evaluate the surveillance system used in this study. Flexibility refers to the system's ability to accommodate new diseases and health conditions (CDC, 2001). The influenza immunization surveillance system used in this study was designed to collect influenza immunization data. However, this system can capture information on other vaccines, such as the pnemococcal vaccine, if seen as important by the Department of Health and Community Services.

Another criterion used to evaluate the surveillance system used in this study is representativeness. A surveillance system is representative if the data obtained from the system actually reflects that of the target population (CDC, 2001). An ideal influenza immunization surveillance system would obtain data that is representative of the entire population. There are two methods of obtaining a representative surveillance system. One method is to have $100 \%$ participation from health care professionals who administer the vaccine, and the second method is to obtain a sample of health care professionals that administer vaccine to a population that is representative of the actual target population. Study results are discussed in the context of the first approach.

The final criterion is timeliness. The timeliness of a surveillance system should be evaluated in terms of the availability of information to identify trends, outbreaks, or the effects of control efforts (CDC, 2001). It is important to receive the influenza immunization data as early in the influenza season as possible, since data specific to the season would help guide interventions for increased uptake in the same influenza season. This study's results will be discussed along with the study conducted by 
Macdonald et al. (1996) and with some reference to the Manitoba Health (2000) study. These studies were conducted on influenza immunization surveillance in the community setting and were previously discussed in Chapter 2. This present study, along with these studies, can increase the understanding of how to create an influenza immunization surveillance system that is effective and acceptable.

\section{Overall Sensitivity}

The sensitivity of a surveillance system can be defined as the system's ability to detect the proportion of cases of a disease or health condition, as well as the system's ability to detect epidemics (CDC, 2001). When determining the sensitivity of a surveillance system one has to consider the system's ability to measure the proportion of cases detected by the surveillance system. Since a purpose of this study was to obtain data on everyone who received the influenza vaccine, the ability of the surveillance system to account for the proportion of influenza vaccine doses administered would determine the system's overall sensitivity.

To discover the overall sensitivity of the surveillance system used in this study, the proportion of administered doses documented was determined. The sensitivity of the surveillance system used in this study was fair due to the system's ability to account for $57.5 \%$ of all vaccine that had been distributed to health care professionals. The sensitivity was higher (74.3\%) when looking at the doses of influenza vaccine accounted for by only participating health care professionals, since there were $13.6 \%$ of health care 
professionals that did not participate. This finding is disappointing, since the surveillance system should account for $100 \%$ of the doses distributed. However, when comparing this surveillance system to the government surveillance system used during the same season by two of the other health regions of the province, the overall sensitivity of the surveillance system used in this study was much better. The government surveillance system in these regions accounted for $25-30 \%$ and $30 \%$ of the influenza vaccine distributed.

The greater overall sensitivity of the surveillance system used in this study $(57.5 \%)$ may be attributed to the structured system that was put in place prior to initiating the influenza immunization system. All health care professionals were provided with a letter explaining the surveillance system prior to receiving their surveillance forms and all forms were asked to be returned to the $\mathrm{MOH}$ once completed. Health care professionals were also reminded to return forms on a monthly basis and were provided with self addressed stamped envelopes to return forms. This made it very easy for health care professionals to return completed forms and reminding them to return forms may have decreased the chance of not returning them. This structured system was not in place in the other health regions and health care professionals were simply sent the government form and asked to return it when influenza immunization clinics were completed. This may be a reason why the sensitivity of two of the other provincial health regions was very low $(25 \%-30 \%$ and $30 \%)$. There was one provincial region who reported accounting for $90 \%$ of the vaccine distributed. This provincial region differed from the 
other regions, as well as this study's region, in that the majority of the vaccine was distributed to community health nurses $(82.4 \%)$. This difference will be discussed later in this section.

The overall sensitivity of the surveillance system used in this study is similar to that stated in the report of the study conducted by Macdonald et al. (1996). Macdonald et al. reported that the health care professionals in their study administered $77.7 \%$ of the doses they had been distributed. Because Macdonald et al. did not include, in their denomiator, the doses distributed to those health care providers who did not participate, the doses documented as administered should be compared to the $74.3 \%$ of doses documented as administered + returned by participating health care professionals in this study. The supportive structure that was in place in both this study and the study by Macdonald et al. may be a reason why both studies have comparable systems in terms of their sensitivity. In addition, the results of the study by Macdonald et al. showed the sensitivity of a surveillance system after a pilot project had already been implemented. The sensitivity of a system similar to the one used in this study may be greater if it is tried again with recommendations added from the feedback received from the health care professionals who used the system.

Feedback was also received from health care professionals to determine if they were able to account for the vaccine they administered by using the surveillance forms. This data provides an indicator of the system's overall sensitivity. All of the nurses and the majority of physicians $(79.3 \%)$ felt they were able to account for most of the vaccine 
they administered by using the surveillance form they had been provided. The six physicians who stated they were not able to account for all the vaccine administered did not feel it was because of the nature of the form. They did not state that the form did not allow them to account for the vaccine administered. Instead, it was mainly because they forgot to complete the forms on several occasions. This feedback supports the conclusion that health care professionals felt they were able to account for most of the vaccine they had administered by using the surveillance form.

The sensitivity of the surveillance system differed depending on whether the forms were completed by community health nurses or physicians. In this study community health nurses documented $95.5 \%$ of the doses they had been distributed, however they only received $3.5 \%$ of the overall doses distributed. The other Health and Community Services Regions of the province that provided feedback on the information obtained from their surveillance initiative showed that the region that had the highest proportion of nurses administering vaccine $(84.2 \%)$, had the highest proportion of forms returned and doses of influenza vaccine accounted for (90\%). In addition, the public health nurses in the study by Macdonald et al. (1996) received 23.7\% of the doses distributed and administered and documented the highest proportion of the doses distributed, $86.9 \%$.

In comparison, physicians in this study documented $65.0 \%$ of the doses of influenza vaccine they received, which was slightly higher than the proportion reported in the study by Macdonald et al. (1996) in which physicians documented $52.3 \%$ of doses 
they had been distributed. In this study, the physicians received $96.5 \%$ of the doses distributed. In the study by Macdonald et al. physicians received $61.9 \%$ of the doses distributed. The surveillance systems in this study and in the study by Macdonald et al. were less sensitive when used by physicians.

The reason why the surveillance system was seen as highly sensitive when used by community health nurses may be due to the fact that community health nurses in the Eastern Region of Health and Community Services are familiar with collecting immunization data since they provide over $80 \%$ of childhood immunizations in the region (personal communication, Patricia Heath, Communicable Disease Control Coordinator Eastern Region, November 24, 2002). If there had been more community health nurses administering influenza vaccine in this study, there may have been a greater proportion of doses documented on the surveillance forms as seen in the study by Macdonald et al. (1996) and in the other provincial region. This supports increasing the use of nurses in administering influenza vaccine. However, it is important to remember that the community health nurses in this study only received $3.5 \%$ of all the vaccine distributed in the 2001-2002 season. It is possible that if community health nurses administer more vaccine in future seasons, because of their increased workload they may not document such a high proportion $(95.5 \%)$ of vaccine administered on the surveillance forms. However, the data from the region that had a high proportion of nurses administering vaccine (90\%), suggests that community health nurses were still able to account for the vaccine they administered. 


\section{Sensitivity by Surveillance Form}

The influenza immunization surveillance forms were compared to determine which form had the highest sensitivity for collecting influenza immunization surveillance data. There were no previous studies found that compared surveillance forms. In comparing the three forms for doses administered, this study revealed that health care professionals who completed the government form documented the highest proportion of influenza vaccine (86.0\%), compared to the tally form $(62.7 \%)$ and the individual form (58.1\%). By using Fisher's Exact Test, the differences in the proportion of vaccine documented between the government and tally form, government and individual form, and the tally and individual form were all found to be significant $(\mathrm{p}<0.0005)$. This shows that health care professionals who completed the government form documented a statistically significantly higher proportion of vaccine, than those who completed the individual and tally forms. Therefore the government form had the highest sensitivity for accounting for the doses of vaccine administered.

Similarly, significantly higher proportions were accounted for when looking at the administered + returned vaccine. Health care professionals who received the government form again documented the highest proportion of influenza vaccine $(86.0 \%)$, compared to the individual form (76.1\%) and the tally form $(64.4 \%)(\mathrm{p}<0.0005)$. Despite the fact that the individual form had a higher proportion of vaccine administered + returned than the tally form, the results were skewed because there were only three physician's offices who returned vaccine and the majority of doses (710) were returned 
by one physician who had received the individual form.

In summary, when comparing forms to determine which form had the highest sensitivity for accounting for the number of doses administered as well as the number of doses administered + returned, the government form was the best. However, the tally form accounted for a significantly higher proportion of documented vaccine than the individual form $(\mathrm{p}<0.0005)$. Therefore, this form showed a higher sensitivity than the individual form in accounting for the number of doses of influenza vaccine administered.

\section{Vaccine Wastage}

Although this study focussed on accounting for the number of doses of influenza vaccine administered, this study's results showed there were 5,656 doses of influenza vaccine unaccounted for. There were only 830 doses returned from three physicians' offices, of which 710 were returned from one physician's office. With such a small proportion of vaccine returned and a large number of vaccine doses unaccounted for $(5,656)$ it is unknown if this vaccine was unused, or if it was administered and not documented as such.

It is, therefore, very important to determine ways to account for unused vaccine. Whether health care professionals complete a separate form at the end of the season stating the amount of vaccine unused and discarded, or are asked to return unused vaccine in order to receive vaccine in the next season, a system is required to track 
unused vaccine. Determining the amount of unused influenza vaccine would also help determine the sensitivity of future influenza immunization surveillance systems, because if the vaccine that was unaccounted for was administered and not documented as administered the sensitivity would be as calculated $57.5 \%$. However, if all the unaccounted for vaccine was actually unused the sensitivity of the system would be $100 \%$, because the forms would have accounted for all of the administered influenza vaccine. It was not anticipated prior to the initiation of the study that health care professionals would not return unused vaccine, since there was no data available from previous seasons to indicate that this would occur. Therefore, a structured method of accounting for unused vaccine had not been implemented. A method to track unused vaccine would have been useful when evaluating the influenza immunization surveillance system used. It would, therefore, be beneficial to implement a structured method to track unused vaccine in future influenza immunization surveillance initiatives.

\section{Summary of Sensitivity}

To promote influenza immunization surveillance in the future it is important to choose the most appropriate surveillance system. The overall sensitivity of the surveillance system used in this study was fair because it was able to account for $57.5 \%$ of the doses distributed. Those who participated accounted for $74.3 \%$ of the vaccine doses they had been distributed. Therefore, if there was higher participation in future influenza immunization surveillance initiatives, there would be at least $74.3 \%$ of vaccine 
accounted for. It is also important to remember that the actual sensitivity of the system was difficult to determine because there were 5,656 doses of influenza vaccine that were unaccounted for. This shows that there needs to be a greater emphasis placed on accounting for unused vaccine in future influenza immunization surveillance initiatives, which would also improve sensitivity.

The sensitivity of the surveillance system varied depending on who completed the surveillance forms. With a greater proportion of doses documented by community health nurses, the sensitivity of the surveillance system was higher than when the system was used by physicians. This finding supports the use of community health nurses to administer more influenza vaccine. One must, however, take into account that the community health nurses in this study only received $3.5 \%$ of the total distributed vaccine. If community health nurses administer more influenza vaccine in the future it may increase their workload, therefore, strategies may need to be implemented to ensure that they can continue to account for as much of the vaccine doses as they did in this study. Health care professionals who completed the government form accounted for a greater proportion of doses administered and administered + returned than those who completed the individual and tally forms. This finding shows that the government form was the most sensitive form, suggesting that it would be the best form to use if sensitivity were the only criterion to consider in finalizing decisions about influenza immunization surveillance. 


\section{Positive Predictive Value}

Positive predictive value, primarily used in screening tests, determines the proportion of persons identified as being cases, who actually do have the condition under surveillance (CDC, 2001). The positive predicative value of this surveillance system was considered as the proportion of individuals documented as receiving influenza vaccine that actually received the vaccine. In comparison to sensitivity, which refers to the system's ability to account for the proportion of influenza vaccine doses administered and those administered + returned. For the surveillance system used in this study, the positive predictive value was difficult to determine, because the study was not set up to obtain the data required to validate that a person to whom vaccine was administered and documented actually received vaccine.

The government form may have a lower positive predictive value than the individual and tally forms because of the nature of the form. The government form required health care professionals to place a tick on the form and then tally up the total number of individuals receiving vaccine on the form at the end of each clinic. In comparison, the tally and individual forms required health care professionals to provide specific information about each vaccine recipient. Therefore, those who completed the government form may have been more likely to indicate that an individual received the influenza vaccine when they actually did not receive it. There was one physician who completed the government form and documented administering 20 more doses than this particular physician actually received, however other than this incident the accuracy of 
the data received from the government form was difficult to determine. This was the only incident of an inaccurate count. Overall the accuracy of the data was difficult to determine.

Overall, there was no reason to believe that anyone would complete a form on an individual, or indicate that an individual received influenza vaccine, if the vaccine was not given. Therefore, there is no reason to believe that the surveillance system used in this study has a weak positive predictive value.

\section{Simplicity and Acceptability}

Determining if a surveillance system is simple and acceptable to those who use it are two important factors to look at when evaluating a surveillance system (Centre for Disease Control, 1988). The simplicity of a surveillance system refers to both its structure and ease of operation. A surveillance system should be as simple as possible while still meeting its overall purpose. A simple surveillance system will lead to a system that is better accepted by those who have to use it, which will in turn increase the willingness of individuals to participate. It was, therefore, important to determine if the surveillance forms used in this study were seen as a simple method to collect influenza immunization data by the health care professionals who administered influenza vaccine. If health care professionals viewed the surveillance forms as simple to complete they would be more likely to accept the surveillance forms, and the same surveillance forms could be used in future seasons. If the surveillance forms were complicated to use, health 
care professionals would probably be less likely to use them in the future.

As a way to determine whether or not health care professionals accepted the surveillance forms used in this study, they were asked to provide feedback on the forms. The other studies that had been conducted on influenza immunization in the community did not obtain feedback from the participants about the surveillance initiative (Macdonald et al.1996; Manitoba Health, 2000). All of the health care professionals that were sent surveillance forms were contacted after surveillance forms were returned to provide feedback on the forms. All of the community health nurses that were contacted provided feedback and $60.7 \%$ of physicians provided feedback.

The majority of the health care professionals who provided feedback ( $86.6 \%$ of community health nurses and $72.4 \%$ of physicians) revealed that they would like to see the same surveillance form used again. There were a total of 10 health care professionals who would have liked to see the surveillance form changed, 8 of whom completed the individual form. The main reason why they wanted to change the form was because there was too much paper by having to complete one sheet per patient, and so they would have liked to see the form condensed into a tally style form. Therefore, if these health care professionals had received the tally form, they may not have had a problem with using the same form in the future. This feedback supports the use of a tally form in future influenza immunization surveillance initiatives.

Health care professionals in this study were also asked to provide feedback on their opinion of influenza immunization surveillance in general. All of the community 
health nurses felt that influenza immunization surveillance is a good idea. Community health nurses play a key role in immunization surveillance since they provide and document immunizations on a regular basis (Zahner,1999). Community health nurses use the data they collect from immunization surveillance to plan and evaluate immunization programs. A few of the nurses elaborated on their feedback and provided insight regarding the connection between influenza immunization surveillance and preparing for pandemic influenza. They stated that pandemic influenza planning is ongoing and completing influenza immunization surveillance forms would help determine who is and is not receiving the vaccine. This would help nurses focus campaigns on those who do not receive the vaccine to try and increase uptake before a pandemic strikes. By analysing the nurses' feedback regarding surveillance, one can easily see that nurses view surveillance as an essential method to collect and evaluate immunization data.

The majority (77.4\%) of all physicians who provided feedback also felt the influenza immunization surveillance was a good idea. Some of the physicians stated that they have been collecting influenza immunization data for years. They had not been forwarding the information on to the provincial level, but used the information to compare their individual practice influenza vaccination rates from year to year. Influenza immunization surveillance is obviously not something new to some physicians in the Eastern Region of the province.

There were $24.1 \%$ of physicians who did not feel influenza immunization 
surveillance was of any benefit. They felt that surveillance was not important and they did not have time to collect this data. Those physicians who did not feel influenza immunization surveillance was important all felt that they did not want to see the same surveillance form used again. They stated that they would like to have a form with fewer items to complete on each patient, and those who completed the individual form would have liked a tally form type or a form that would not take so much time to complete. These physicians did not feel surveillance was important and therefore, may not have agreed to use any form in the future. It is important to educate physicians about the importance of influenza immunization surveillance as a way to help increase their participation in future surveillance initiatives.

Time was not seen as a great concern for those who completed the surveillance forms, since the forms did not take much time for health care professionals to complete. Health care professionals estimated that it took about one minute to complete the information on each person vaccinated, with the maximum amount of time spent to complete the surveillance forms being 2.75 hours a week for approximately two weeks a year. Because it only took one minute to complete each form, time would probably not be a factor as to why health care professionals would not accept the surveillance forms in future initiatives.

Overall, the feedback received from health care professionals showed that using influenza immunization surveillance forms was a simple and acceptable way to collect influenza immunization surveillance data. The feedback also showed that of the 
individual and tally form, the tally form was the form they would like to see used again. Those who completed the individual form and did not like it wanted the form to be in the format of a tally sheet. Therefore, the tally form was the most accepted form of the two and would be the most appropriate form to use in future seasons if using this criteria only. All of the nurses were strongly in favour of influenza immunization surveillance and indicated that they understood the importance of such an initiative. The majority of physicians felt influenza immunization surveillance was important, however, a $24.1 \%$ could not see the importance of surveillance and would not like to see such an initiative added to their current workload, even though time was not a major issue for health care professionals when completing the forms. It only took a minute to complete the information on each individual which made the forms that much more acceptable to health care professionals. With increased awareness of the importance of influenza immunization surveillance, these physicians may become more enthusiastic about participating in future surveillance initiatives.

\section{Complete, Accurate and Useful Data}

It is important to determine the completeness of the data received from the returned surveillance forms, since incomplete data would influence the sensitivity of the surveillance system used. The more information completed by health care professionals, the better the surveillance system is in determining the proportion of individuals vaccinated and developing a profile of recipients. 
When completing the government form, health care professionals were only expected to tally up the doses administered after each clinic and fill in the number on the form. Therefore, there were no missing data due to the nature of the government form. Health care professionals that completed the individual and tally forms had six items to complete and there were similar proportions of data missing from both the individual and tally forms, with $10.6 \%$ and $15.0 \%$, respectively, having at least one piece of data missing. The tally and individual forms had a space for health care professionals to document the reason why individuals received the influenza vaccine. This item was the most important since it provided numerator data for those vaccine eligible groups who received influenza vaccine. There was $99.6 \%$ of this information provided by health care professional who completed the tally form and $99 \%$ by those who completed the individual form. Due to the fact that these surveillance forms had such a small amount of data missing from this item, it shows that health care professionals who completed both forms were effective in collecting data on those individuals who received the influenza vaccine.

The question related to whether the patient received pneumococcal vaccine had the most missing data. However, the percentage of data missing from this question was still minimal with $7.4 \%$ of entries missing from tally forms and $6.1 \%$ missing from individual forms. There are several reasons why entries related to pnemoccocal vaccine were possibly missing. One reason may be due to the fact that, unlike the other questions, this information may not have been easily retrieved from patient files because 
pneumococcal vaccine is not provided every year like the influenza vaccine. After the surveillance initiative began, a call was received from one physician who wanted more information about the pneumococcal vaccine. This physician felt that he did not know enough about the vaccine and when it should be offered to patients. He said that he was not the only physician he knew that would like more information on the vaccine. There is information sent out with the pneumoccocal vaccine every year and it is important that physicians read this information. Because physicians may not read the information provided to them, they may not know when to offer the pneumococcal vaccine to their patients. This may be another reason why there was more data missing on this question than any other question, because physicians who do not know when to offer the vaccine may avoid asking the patient if they had received the vaccine before, due to fear of the patient asking questions about the vaccine that they may not be able to answer. A third possible explanation for the missing data may be that individuals may not remember receiving the vaccine if they were asked about past pneumococcal vaccination.

The accuracy of the data relates to whether the data obtained from the influenza immunization surveillance forms truly reflect that of each individual who received the influenza vaccine. The accuracy of the data obtained from the surveillance forms i.e. was the information true is a different issue than the completeness of the data obtained i.e. was the information documented. However, the surveillance system's ability to provide accurate data is also important to consider. Accuracy of the data obtained is an important component because inaccurate data leads to an inaccurate estimate of those individuals in 
the population who were actually vaccinated. The individual and tally forms required health care professionals to complete more specific data on the recipients of the influenza vaccine than the government form required. These forms had a space provided to indicate the patient's date of birth and space to indicate the reason why the patient was vaccinated. Four percent $(78$ of 1,950$)$ of health care professionals identified age as the only reason why their patient received vaccine despite the fact that the individual receiving the vaccine was under 65 years of age. This was the only example of possible inaccurate data. It can only be assumed that the information provided by health care professionals was accurate since most health care professionals have a good knowledge of their patients and why they are receiving the vaccine. Therefore, other than the $4.0 \%$ discrepancy that was noted between the actual age being under 65 years and age identified as a reason for vaccination, there was no reason to doubt the accuracy of the information provided on the surveillance forms.

It is important to determine which surveillance form provided the most useful influenza immunization surveillance data. The most useful information obtained from the surveillance forms would be the information regarding who received the influenza vaccine. Because there are multiple target groups who should receive influenza vaccine, it is important to ask questions on surveillance forms to distinguish between these target groups. The tally forms and individual forms had six questions which health care providers were expected to complete, whereas the government form only looked at whether the recipient was under or over 65 years of age. The data from the tally and 
individual forms allows one to summarize the reasons why individuals received the influenza vaccine. This information can be very useful if the appropriate denominator data is available, since it provides good numerator data which helps in determining the proportion of individuals in certain target groups that are receiving influenza vaccine.

When reviewing the present tally and individual forms, there were two questions that were not related to the specific purpose of this surveillance system and could possibly be removed from the forms if they were to be used again in future influenza immunization surveillance initiatives. The recipient's gender could be removed from the form since it is not a risk factor for contracting the influenza virus and there is at present no difference in the methods used to promote vaccine uptake in males and females. However, if significantly more females receive the influenza vaccine than males, or vice versa, it would be important to add gender-specific strategies that target those who do not receive the vaccine in future influenza immunization promotional campaigns. Since gender is not time consuming to record the decision to keep or delete this question on future surveillance forms will have to be made by the Department of Health and Community Services if they deem it important to know if a gender specific difference exists. The recipient's specific date of birth could also be removed and replaced with an under 65 years and a 65 years of age and older category; the form would be easier to complete and the required information would still be obtained. It is important to keep the date the influenza vaccine was administered on the form because it may be helpful in the future to track when most of the vaccine was administered to guide interventions to 
increase vaccine uptake for that season.

The reasons why individuals received the influenza vaccine could be merged into fewer categories. Health care professionals could remain as a separate category since this is a group of individuals that has denominator data available. Therefore a vaccination rate for this group could be determined if future surveillance initiatives included institutions. Diabetes could also remain as a separate category because there is denominator data available for this population. The other chronic diseases such as cardiac, pulmonary, renal disease and cancer could all be merged as one category called "chronic disease". Age could remain as a reason and an "other" category would remain so health care professionals could write in the reason why the vaccine was provided if it did not fit the other categories. The question of whether the patient received the influenza vaccine in the past should remain on the form. This question provides information regarding whether there is an increase in new vaccine recipients each year, or if health care professionals are continuously vaccinating the same individuals each year. In addition, the individual and tally forms had a question related to whether or not the patient received pneumococcal vaccine in the past. Retaining this question is discussed in the next section on flexibility.

Overall, with respect to this criteria, the tally and individual forms were more useful than the government form. The completeness and accuracy of the information obtained was not a concern from either one of the three forms. Modifications could be made to further simplify the tally and individual forms without reducing the usefulness of 
the data. This finding shows that the individual and tally forms provided the most useful information, suggesting that these would be the best forms to select if usefulness were the only criterion to consider in finalizing decisions about influenza immunization surveillance.

\section{Flexibility}

The criterion of flexibility, the system's ability to accommodate new diseases and health conditions, can be seen in the influenza immunization surveillance system used in this study (CDC, 2001). By including an extra question on the surveillance form, this system can easily accommodate other vaccines such as the pnemococcal vaccine.

There are arguments both for and against retaining the question relating to whether or not the individual received pneumococcal vaccine in the past, which was presented on the tally and individual forms. The question can be removed if the future surveillance system's only objective is to collect influenza immunization data. Doing so would condense the form to have fewer questions and possibly reduce the amount of missing data.

There are a number of benefits to keeping this question on the surveillance form. Keeping this one extra question is a simplistic way to track two vaccines with one surveillance form. In addition, pneumococcal vaccine surveillance can be tied in with influenza immunization surveillance as a way to flag pneumoccocal vaccination as a topic for discussion between health care professionals and their patients. If patients see 
this question on the form they may ask their health care professional about the vaccine and if they should receive it. As well, health care professionals may bring up pneumococcal vaccination to their patients more frequently. Because the results of this study showed that $47.7 \%$ of those 65 years of age and older did not receive pneumococcal vaccine in the past, there is still work needed on promoting the administration of pneumococcal vaccine. Therefore, it may be important to keep this question on future surveillance forms as a way to promote pneumoccoal vaccine administration in the future. Ultimately the decision to delete or keep this question on future surveillance forms will have to be made by the Department of Health and Community Services when developing future surveillance initiatives.

\section{Representativeness}

In order to receive good quality influenza immunization data, the information obtained from returned surveillance forms must be representative of the recipient population. A surveillance system is representative if the data obtained from the system actually reflects that of the target population. An ideal way to have an influenza immunization system that obtains data that is representative of the entire population is to have $100 \%$ participation from health care professionals who administer the vaccine. Another way to obtain a representative sample is to select health care professionals that provide influenza immunization to a sample of patients who are representative of all those who receive the vaccine, and have these health care professionals collect influenza 
immunization data. Without high participation from health care professionals, or a true random sample of the target population, the use of influenza immunization surveillance forms would not be an effective way to collect data that is representative of the population who receive the influenza vaccine, since a portion of the population would be left out.

The overall participation in this study was $91.2 \%$ with $100 \%$ participation by community health nurses and $82.4 \%$ participation by physicians. Similar participation was seen in the study by Macdonald et al. (1996) with an overall participation of $82.7 \%$ and $100 \%$ participation by public health nurses and $71.9 \%$ by physicians. Therefore, in comparing these results, community health nurses have a higher participation rate in carrying out influenza immunization surveillance.

There was $100 \%$ participation by community health nurses in this study, as well as in the study by Macdonald et al. (1996). Community health nurses in the Eastern Region of Newfoundland and Labrador are familiar with participating in immunization surveillance since they provide over $80 \%$ of the childhood immunizations in the region (personal communication, Patricia Heath, Communicable Disease Control Coordinator Eastern Region, November 24, 2002). When nurses provide the immunizations to children, they are required to collect and document yearly immunization surveillance data and send this information to the Communicable Disease Control Coordinator for data entry. Therefore, the process of collecting surveillance data is a very familiar one to community health nurses, and all of the community health nurses who participated in this 
study felt that influenza immunization surveillance was a good idea, this may be the reasons why there was $100 \%$ participation from the nurses in this study. With a high participation rate, community health nurses are effective in collecting numerator data for those to whom they administer the influenza vaccine. Therefore, if there were more nurses administering influenza vaccine there may be a higher overall participation in influenza immunization surveillance which would in turn lead to more accurate numerator data.

The participation by physicians in this study and in the study by Macdonald et al. (1996) was less than the $100 \%$ participation by nurses. Of the physicians who were distributed vaccine in this study, $82.4 \%$ completed and returned surveillance forms and $71.9 \%$ of the physicians in the study by Macdonald et al. participated by returning forms. The physicians in this study were asked for feedback as to why they did not participate. Those who provided feedback stated that they did not receive the form on time and they misunderstood how to complete surveillance forms. Therefore, if physicians received the forms before surveillance began and better understood how the surveillance forms should be completed there may be an increase in participation by physicians in future surveillance initiatives.

This present study was the first influenza immunization surveillance project of this nature in Newfoundland and Labrador and, therefore, showed high participation for the first time for such an initiative. If a similar surveillance initiative took place again there may be a higher participation rate as seen in the study by Macdonald et al. In their 
study, the overall response in the 1993 pilot project was $47 \%$ and increased to $82.7 \%$ when the surveillance initiative was tried again in the most recent study.

This study was the only study that compared three different surveillance forms. If the government form was not included in this study, and the study was carried out as it was originally planned, the participation rate would have been $62.7 \%$. This rate is still relatively high for the first time for such an initiative. There was very little difference noted in participation by physicians who originally received the individual, $52.6 \%$ (10/19) and tally, 47.4\% (9/19) forms. The government form was sent to those who refused to complete the individual and tally form. Physicians were not forced to complete the government form but were encouraged to complete it if they had refused to complete the form they had been originally sent.

This study achieved $91.2 \%$ participation from health care professionals who administer influenza vaccine. This participation rate suggests that it is possible to improve participation in the future influenza immunization surveillance initiatives and get close to $100 \%$ particiaption, which would ensure representativeness of the data. The alternative would be to try and obtain a representative sample of patients. This could be done by selecting health care professionals in the region that provide influenza immunization to a population that is representative of those who receive the influenza vaccine, and have these health care professionals collect influenza immunization surveillance data. A random selection of all health care professionals who administer influenza vaccine or using volunteers may be two other alternatives. The physicians who 
are already involved in the influenza surveillance or those who have already been carrying out influenza immunization surveillance and keeping their own statistics for previous seasons, may be two examples of physicians who would be willing to volunteer. In either case, it would be still necessary to be sure of the representativeness of the patients they see.

Generalizability of the patients of health care professionals who volunteer to participate in research or surveillance has not been well described. Green, Miller, Reed, Iverson, and Bailey (1993) evaluated the patients and practices of family physicians in a national practice-based research network to understand whether results from practicebased research networks are likely to be relevant to other practising clinicians. The results of their study showed that overall, the two samples differed with respect to demographics and characteristics of patients, while problems, diagnoses, services, disposition, and time spent with patients were similar. Despite the fact that this study showed that practice-based research networks are sufficiently representative of family practice, there is not enough evidence to show that specific family practice physician clinics in areas of this province are representative of those in the entire region.

Therefore, prior to selecting the health care professionals, the population served by the individual practices would need to be determined.

One method to determine the patient population served by family practice physicians is to review the practices' client files and determine the type of patients seen in that particular area. This would provide the information required to select those 
physicians who serve a population that is truly representative of individuals who receive influenza immunization. In order to obtain this information a separate study would be required, since the files would have to be reviewed before the appropriate physicians were recruited to conduct influenza immunization surveillance. Without this information it would be difficult to determine how to select practices or if the practices of physicians who volunteer to participate would be representative of those who receive influenza immunization.

In summary, this study showed that because of the high participation from health care providers $(91.2 \%)$, the information received from the surveillance forms was fairly representative of those who received influenza vaccine. The results of this study and the study by Macdonald et al. (1996) show that there are several ways to help increase health care professionals' participation in influenza immunization surveillance. Determining ways to increase participation to close to $100 \%$ will lead to a greater representation of those who receive the influenza vaccine in future seasons. It may be easier to try the strategies recommended by this study's conclusion and improve participation than to try and find health care professionals whose patients are representative of the target population.

\section{Timeliness}

The timeliness of a surveillance system should be evaluated in terms of the availability of information to identify trends, outbreaks, or the effects of control efforts. 
The need for a rapid response in a surveillance system depends on the nature of the problem under surveillance and the objectives of the system. It is important to receive the influenza immunization data as early in the influenza season as possible, since data specific to that year could help guide interventions to increase vaccine uptake during that particular influenza season. There was no data collected in this study which related to when the forms were actually returned. It is therefore not possible to determine if the forms were returned in a timely manner.

The majority of the vaccine was known to have been administered in October and November of the 2001-2002 influenza season. It would, therefore, be beneficial to have health care professionals return their surveillance forms at the end of every month and then the majority of surveillance forms would be returned by the end of the first two months of the influenza season. Ensuring a timely return of surveillance forms would be an important component to add to future influenza immunization surveillance systems.

\section{Conclusions Regarding Improving Influenza Immunization Surveillance} Influenza immunization surveillance in general was seen as a good idea by health care professionals in the Eastern Region. All of the community health nurses were strongly in favour and understood the importance of influenza immunization surveillance. The majority of physicians felt influenza immunization surveillance was important, with only a few who could not see the importance of surveillance and who would not like to see such an initiative added to their current workload. Therefore, it is 
important to educate these physicians about the importance of influenza immunization surveillance to help increase their willingness to participate in future surveillance initiatives. Overall, however, the findings from this study support continuing with the influenza immunization surveillance system initiated for this study with three changes.

The government form, although it accounted for the highest proportion of documented doses, did not provide information about reasons why individuals received the influenza vaccine. In comparing the tally and the individual forms, those who completed the tally form accounted for a statistically significant higher proportion of administered vaccine than those who completed the individual form $(\mathrm{p}<0.0005)$. Both forms provided useful information regarding why individuals received vaccine and one form had no more missing data than the other. Those who completed the individual form were less accepting of the form, since they felt there was too much paper used and they would have liked to see the form as a tally format. Health care professionals also wanted to see fewer categories on the surveillance forms. Therefore, to increase acceptance of the surveillance system in future seasons, the tally form should be used with the modifications previously described.

In addition, to obtain influenza immunization data, it is important that the Department of Health and Community Services develop a method and policies as necessary of surveillance to account for unused vaccine in future surveillance initiatives. Accounting for unused vaccine will help determine the true sensitivity of the surveillance system used, as well as provide a true picture of those target groups who receive 
influenza vaccine. The Department of Health and Community Services must also be willing to provide human resources to collect and analyse the data which is obtained from an influenza immunization surveillance system. These human resources, along with an influenza immunization surveillance system that has been evaluated as being effective in meeting its overall objectives, will aid in collecting good quality data in future influenza immunization seasons.

Finally, to increase the representativeness of the surveillance system used in this study, it is important to try to increase participation to have all health care professionals who administer influenza vaccine provide influenza immunization surveillance data. This may be easier to achieve than selecting health care professionals in the region who administer the influenza vaccine to a population that is representative of those who receive the vaccine and have them collect influenza immunization data. Community health nurses had a higher participation rate than physicians which supports having more influenza vaccine administered by community health nurses as a way to increase the participation rates of future influenza immunization surveillance systems. Increasing the amount of vaccine administered by community health nurses could only occur if there are extra human resources available to help with the increased workload that would result from increasing the number of influenza vaccines that they would have to administer. Providing information, support, and resources to all health care professionals should help increase participation as well as the overall effectiveness of the surveillance system. 


\section{Improving Surveillance of Target Groups}

The focus of this study has been on collecting numerator data. However, to determine the proportion of vaccine eligible groups who receive influenza vaccine, one must not lose sight of the importance of denominator data. In order to calculate this proportion, the number of doses of influenza vaccine administered and to whom they were administered is required (numerator data) as well as the number of individuals eligible for the vaccine (denominator data). The proportion of vaccine eligible groups who received influenza vaccine was difficult to determine from the results of this study as there were problems with both numerator and denominator data.

The first problem with numerator data was that there were nine physicians who did not participate. These physicians received 3,040 doses of influenza vaccine. This vaccine could not be included in the numerator which would alter the calculated proportions. Another problem was that the government form only provided information on whether or not individuals who received vaccine were under 65 or 65 years of age and older. Age was the only item that all three forms had in common. When calculating the proportion of the other target groups vaccinated, such as diabetics, individuals with cardiac and pulmonary disorders, the 2,123 doses administered to individuals by health care professionals who completed the government form could not be included because the required information about health conditions was not available. Both of these problems contribute to an error when calculating the total proportion of groups vaccinated because a total of 5,163 doses were not included in the numerator. It is important to choose an 
influenza immunization surveillance form that provides information on why individuals receive influenza immunization.

Another problem with calculating the proportion of target groups vaccinated lies with the lack of appropriate denominator data. This particular study was carried out in the 2001-2002 season. The last census data published for Health and Community Services, Eastern Region, of the province of Newfoundland and Labrador was in 1996. There will be data available for the 2001 year, however, it was not available when this data was required. If the data were available earlier, or if there were no time constraints on submitting the findings of this study, the researcher could have waited and used the 2001 census data to calculate the proportion of individuals 65 years and older who received influenza vaccine. The denominator data available from the 1996 census data may not be the same as the data for 2001 . Therefore, the actual proportions may be higher or lower than those calculated with the 1996 data, depending on if there was an increase or decrease in the target population.

There is not a lot of data available on the number of individuals living in the community with chronic diseases. The data is even more difficult to collect when the data needs to be broken down into provincial regions. Data on the number of individuals living in the community with diabetes was available for this study.

Denominator data for cardiac and pulmonary disorders and cancer was only available through hospital separation data. This denominator data would not be appropriate because it would not be representative of the individuals who received 
vaccine in the community. If this data was used, one must assume that those who received influenza vaccine were hospitalized for their diagnosis which warranted them to receive the vaccine. This was a large assumption to make, therefore, the proportion of individuals from these target groups could not be calculated from the data collected from the surveillance forms. Recommended changes to forms that would include merging categories because data is not available for all chronic conditions. These changes would match the denominator data that is available, which would help determine proportion of these target groups that are vaccinated.

The participation rates in this study were high for the first implementation of an influenza immunization surveillance system. Therefore, the information received from the influenza immunization surveillance forms was fairly representative of those who receive the influenza vaccine. However, with insufficient region specific denominator data on individuals in the region who are living with chronic conditions, it was difficult to determine proportions of individuals in certain target groups who received influenza vaccine in the 2001-2002 season. This study's purpose was to collect numerator data on those who receive the influenza vaccine and not denominator data. However, it would be beneficial to conduct further studies which could focus on determining region specific denominator data for those individuals who live with chronic disease.

\section{Improving Vaccination of Target Groups}

Once numerator and denominator data is improved, the proportion of individuals 
who receive influenza vaccine will be more easily determined. This information can then be used to focus influenza immunization promotional campaigns on those groups who are not receiving influenza immunization. The results of this study indicated that only a small proportion of diabetics (10.7\%) and individuals 65 years of age and over (25\%) residing in the Eastern Region of Newfoundland and Labrador received influenza vaccination in the 2001-2002 season. These low influenza immunization rates show that further research is required to determine why these vaccine eligible groups did not receive vaccination. These groups may require influenza immunization promotional campaigns to help increase vaccine uptake.

Community health nurses should be utilized to help increase influenza immunization rates. Community health nurses can implement influenza immunization promotional campaigns to help increase vaccine uptake by those individuals who currently do not receive influenza vaccination. Community health nurses could also provide structured influenza immunization clinics to these individuals. The community health nurses would complement physicians who presently administer influenza vaccine, since they could administer vaccine to those who previously did not receive influenza vaccination. This would decrease the burden that could potentially be added to physicians' workloads, while expanding the role of the community health nurse in influenza immunization. 


\section{Strengths of the Study}

The main strength of this study was the high participation from health care professionals. The overall participation in this study was $91.2 \%$ which was very high, considering it was the first time influenza immunization surveillance was carried out. There was $100 \%$ participation by community health nurses and $82.4 \%$ by physicians. The surveillance system used in this study was a very simple system in which health care professionals were sent surveillance forms with their vaccine order along with stamped addressed envelopes to return forms. The health care professionals were also sent monthly reminders to return forms. This simplistic system as well as health care professionals' acceptance of the system, all helped increase their participation. With high participation from health care professionals who administered the influenza vaccine, the information received from the surveillance system used in this study should be fairly representative of those who received influenza vaccine.

Another strength of this study was that it compared three surveillance forms and obtained feedback from health care professionals about the surveillance forms. There were no other studies found in the literature on influenza immunization in the community that compared surveillance methods, nor were there any studies that obtained feedback from health care professionals. Comparing the forms to determine which form provided the most useful information helped in deciding which form was the best form to recommend for future surveillance initiatives. By obtaining feedback from those health care professionals who administered influenza vaccine, it helped determine which form 
was most accepted and it also provided an opportunity for health care professionals to give their opinions on the surveillance system used.

\section{Limitations of the Study}

There was one key limitation of this study. There were 5,656 doses of influenza vaccine unaccounted for and there was no system in place to determine if this vaccine was actually wasted or administered. If the vaccine was wasted and discarded by health care professionals, it would change the sensitivity of the overall surveillance system, because all the vaccine administered would have been accounted for by the surveillance forms used. This would increase the overall sensitivity of the system. Therefore, in future influenza immunization surveillance initiatives it is important to have a mechanism in place to track vaccine that is unaccounted for. This unaccounted for vaccine also lead to incomplete numerator data, as discussed previously in this chapter. Implications of the study's findings are discussed in the following chapter. 


\section{CHAPTER 6}

\section{Implications for Practice, Policy, Education, and Research}

This chapter focuses on the implications of this study's findings. The first section summarizes the discussion of the study's findings found in chapter five. This is followed by several sections which present the implications for community health practice, policy, education, and research. Finally, there is section presenting the overall recommendations of this study.

\section{Summary of Discussion of Study Findings}

The results of this study were discussed in relation to the CDC (2001) guidelines for evaluating a surveillance system. The first criterion used to evaluate the surveillance system was sensitivity. This study's results showed that the overall sensitivity of the surveillance system used was fair, with a possibility of a higher sensitivity if the unaccounted for vaccine was actually unused. The sensitivity differed depending on who completed the form, and which form was completed. When community health nurses utilized the surveillance system, the sensitivity was shown to be higher. Those who completed the government form accounted for statistically significantly more administered and administered + returned vaccine than those who completed the tally and individual forms $(\mathrm{p}<0.0005)$.

The second criterion used to evaluate the surveillance system was the positive 
predictive value. The positive predictive value in this surveillance system was the proportion of individuals documented as receiving influenza vaccine that actually received the vaccine. For the surveillance system used in this study, there was no reason to believe that the positive predictive value was low.

The simplicity and acceptability of the surveillance system was also discussed and the overall feedback from health care professionals showed that the influenza immunization surveillance forms were a simple and acceptable way to collect influenza immunization data. Time was not an issue for health care professionals when completing the forms, and of the individual and tally forms, the tally form was the form they would like to see used again.

The criterion of flexibility, which refers to the system's ability to accommodate new diseases and health conditions, was seen in the influenza immunization surveillance system used in this study (CDC, 2001). The question relating to whether or not the individual received pneumococcal vaccine in the past was on the individual and tally forms. This showed the surveillance system used in this study was flexible since it was able to accommodate other vaccines. Accommodating other vaccines on future surveillance forms would depend on whether or not it was seen as important by the Department of Health and Community Services.

The surveillance forms provided information that was fairly representative of those who received the influenza vaccine, because of the high participation rate $(91.2 \%)$. Community health nurses had a higher participation rate than physicians in this study. 
With such a high participation rate, it would be easier to try to increase participation to $100 \%$ in future influenza immunization surveillance systems than to try and obtain a representative sample of patients.

The final criterion used to evaluate the surveillance system was timeliness. There was no data collected in this study which related to when the forms were actually returned. However, ensuring a timely return of surveillance forms would be an important component to add to future influenza immunization surveillance systems, since collecting data specific to the season would help guide interventions to increase influenza vaccine uptake during the same season.

In conclusion, the system's overall sensitivity may be increased if future influenza immunization systems implement a method to account for unused vaccine. There was no reason to believe the positive predictive value was low. The system that was used was simple and accepted by the majority of those who used it and provided feedback, and the system was shown to be flexible in that it can easily accommodate other vaccines. The system provided fairly representative data, however it can be improved if the participation increases to closer to $100 \%$. There was no data collected on when the forms were returned, however timeliness would be an important criterion to use to evaluate future surveillance systems. This study's findings support continuing with influenza immunization surveillance, using a similar system, while incorporating the recommendations suggested. 


\section{Implications Practice in a Community Setting}

This study has several implications for practice in a community setting. It is important for health care professionals practising in the community to continue with influenza immunization surveillance. The influenza immunization tally form used in this study, with the recommended changes, would be the best surveillance form to use in future influenza immunization surveillance systems.

Another implication for community health practice is to implement a method for health care professionals to account for all unused vaccine at the end of the influenza season. Accounting for the vaccine that was unused during a particular influenza season is important because it would help in providing more accurate numerator data, since the administered vaccine and the administered + returned vaccine would add up to be $100 \%$ of the vaccine distributed. It is recommended that if health care professionals do not return unused vaccine, they should have to complete a form at the end of the season stating the amount of vaccine unused. If they do not do this and the vaccine documented as administered does not add up to the vaccine distributed, they should only receive the amount of vaccine documented as administered in future seasons. In addition, if health care professionals are sending back a lot of unused vaccine, health boards who are receiving the unused vaccine should contact these offices to determine why they had such a large amount of vaccine returned. This monitoring process may help health care professionals become more aware of their actual vaccine usage and needs, leading to more realistic requests for vaccine in the future. This monitoring, coupled with the 
Health and Community Services Board sending health care professionals amounts of vaccine in keeping with previous usage, should reduce the amounts of unused vaccine and the associated costs.

Participation of community health nurses in influenza immunization surveillance was not a problem in this study because there was $100 \%$ participation by community health nurses. The greater issue is to increase community health nurses involvement in influenza vaccine delivery. Community health nurses are familiar with conducting health promotion activities, and should be utilized as a way to help increase influenza immunization. There were two vaccine eligible groups, those 65 years of age and older $(25.0 \%)$ and diabetics $(10.7 \%)$, identified as having low influenza vaccination rates in this study. Community health nurses can implement influenza immunization promotional campaigns and provide influenza immunizations to these individuals, and to others who currently do not receive influenza vaccination. This would not decrease the amount of influenza vaccine that is currently provided by physicians. Instead, the nurses would complement them by administering vaccine to those individuals who previously did not receive influenza vaccination. This would decrease the burden that could potentially be added to physicians' workloads, while expanding the role of the community health nurse in influenza immunization.

In order to utilize more nurses to administer influenza vaccine there must be an increase in resources. Community health nurses would require more human resources to carry out larger influenza immunization clinics. These human resources would only be 
required when influenza vaccination clinics take place.

Finally, it is important to provide feedback to health care professionals of the results of the influenza immunization surveillance data received after each influenza season. Health care professionals will be able to utilize this data as a way to increase influenza immunization of those who did not receive the vaccine. It will also promote the importance of collecting influenza immunization data, and show health care professionals how the data can be utilized.

\section{Implications for Policy}

It is important that the Department of Health and Community Services utilize a mandatory system, with supportive policies, to collect influenza immunization data. With participation being a key component in the collection of influenza immunization data that is representative of those who receive the vaccine, there may be a need for the Department of Heath and Community Services to implement mandatory influenza immunization surveillance. This may be a way to increase participation and lead to a more accurate calculation of the proportion of target groups who received the vaccine.

One of the first initiatives toward developing a national immunization strategy should be to create a reliable, up to date electronic registry system for immunizations (Embree, 2001). This electronic registry would ideally enable vaccine providers to determine which vaccines their patients had actually received and when, regardless of where they had been immunized. These registries would also provide powerful tools to 
monitor the effects of immunization on disease incidence. Despite the fact that electronic recording would be an efficient method to collect influenza immunization data, the feasibility of implementing such a system quickly in this province is low because very few physicians in this province presently have computers and therefore would not be able to record influenza immunization data electronically. Once health care professionals have the technology available to enter this information, computerized recording could become a much more efficient way to carry out future influenza immunization surveillance. The tally form used in this study would be the recommended method of influenza immunization surveillance until the proper technology is available to health care professionals.

Another implication of this study's findings for policy relates to funding. If health care professionals are going to have to carry out influenza immunization surveillance and conduct influenza immunization promotional campaigns, to increase influenza vaccine uptake in those who are not receiving the vaccine, there will be extra resources required. It would, therefore, be important for the Department of Health and Community Services to advocate for more funding so health care professionals can carry out influenza immunization surveillance each year and conduct influenza immunization programs on a larger scale. 


\section{Implications for Education}

Ongoing education about the importance of influenza immunization surveillance is needed for health care professionals who provide influenza vaccination. This may increase health care professionals' participation in influenza immunization surveillance because it will increase their awareness about the importance of the influenza immunization surveillance data they are collecting.

It is important to continue to place an emphasis on immunizations, particularly influenza, in the educational programs of health disciplines that are involved in providing and promoting immunization. This will increase the knowledge nurses and other health care professionals have about immunizations and the important role they play in the administration and collection of immunization surveillance data. Community health nurses who are currently practising may also need some additional information about the influenza vaccine because many nurses may not have had to administer the vaccine in recent years. It would be important to survey community health nurses to determine any continuing education they may need about the influenza vaccine, especially if they began to carry out larger scale influenza immunization clinics.

This study found that most of the data that were missing from the forms were related to whether or not the patient received pneumococcal vaccine in the past. There were several reasons why the majority of data were missing from this question. Physicians may not have read the information provided to them about pneumococcal vaccine and therefore may not know when to offer this vaccine to their patients. 
Physicians who do not know when to offer the vaccine may avoid asking the patient if they had received the vaccine before, due to fear of the patient asking questions about the vaccine that they may not be able to answer. During this study a physician called and stated that he did not know enough about the pneumococcal vaccine to know when it should be offered to patients. Therefore, it is recommended to survey health care professionals in the region as a way to determine those who require continuing education about pneumococcal vaccine. This would identify those who need education so they could be sent the information that they need on the vaccine.

Finally, it is important to educate patients about the importance of receiving yearly influenza vaccinations. This education may take place one on one with health care professionals or through larger influenza immunization promotional campaigns. Patient education will help alleviate any misconceptions patients may have about the influenza vaccine, as well as increase their knowledge about the vaccine and its benefits. This may help increase vaccine uptake in those who presently do not receive the vaccine.

\section{Implications for Research}

The results of this study have suggested further research is required in areas related to vaccine eligible groups and who should administer the influenza vaccine. One of the limitations of this study was difficulty knowing who received the influenza vaccine. Therefore, it is important to increase research in areas that would expand region specific denominator data for specific chronic conditions. Presently there is no region 
specific denominator data available for conditions such as: cancer, pulmonary disorders, cardiac conditions, and renal disease. This data would enable future influenza immunization systems to determine the proportion of target groups vaccinated, and therefore help health care professionals plan ways to target influenza immunization promotional campaigns on those who do not receive the vaccine. In addition, it would be important to understand why certain vaccine eligible groups are not receiving the influenza vaccine. This would help determine methods to increase influenza vaccination in those who presently do not receive the vaccine.

The results of the study showed that community health nurses were effective in collecting surveillance data. Community health nurses are familiar with childhood immunizations and are effective in collecting surveillance data related to these immunizations. Further research is required to determine if community health nurses are as familiar with the influenza vaccine as they are childhood vaccinations. If community health nurses are familiar with the influenza vaccine, it would be important to conduct a study to compare community health nurses to physicians in terms of their knowledge of influenza immunization, storage of the influenza vaccine, and the proportion of unused vaccine. In addition, it would be beneficial to determine the cost of having community health nurses administer influenza vaccine compared to physicians. By conducting such a study, it may help determine which health care professionals are the most appropriate to administer influenza immunizations.

If community health nurses are as effective as physicians in the overall delivery 
of the influenza vaccine it would be beneficial to increase the role of the community health nurses in administering the influenza vaccine. In order to increase community health nurses involvement in vaccine delivery, there must be further research completed on how to increase their involvement.

\section{Overall Conclusions}

In conclusion, it is recommended that the Department of Health and Community Services implement an influenza immunization surveillance system across the province of Newfoundland and Labrador. Influenza immunization forms are the surveillance method of choice and the same influenza immunization surveillance form should be used across all regions of the province. The form should be presented in a tally style format containing 5 questions. The questions on the future tally style form would consist of: date of immunization, whether patient is under 65 years of age or 65 years of age and over, if patient received influenza vaccine last year, if patient received pneumococcal vaccine in past and what was the reason for influenza immunization. The reasons for vaccination should be listed in categories that have denominator data available, until more region specific denominator data becomes available for certain individuals living with chronic diseases such as cardiac and pulmonary disorders.

The forms should be sent to all health care professionals who administer the vaccine or to a sample of health care professionals who administers the vaccine to a population that is representative of influenza vaccine recipients. Both methods would 
help obtain a sample that is representative of those who receive the influenza vaccine. When using a random sample or if distributing forms to all health care professionals, it is important to utilize techniques that help enhance participation. Such techniques should include: using a structured system that explains the surveillance initiatives, returning forms to the $\mathrm{MOH}$, sending out monthly reminders to return forms and obtaining feedback from participants to determine their opinions on the surveillance system used. In addition, it is important to make the surveillance system as simple as possible. The simpler the system, the more acceptable it will be to health care professionals. This will lead to greater participation from health care professionals who administer the influenza vaccine.

Finally, it is important to evaluate the surveillance systems used in future influenza immunization surveillance initiatives. Evaluating the surveillance system in terms of the seven criteria identified by the CDC (2001) guidelines for evaluating a surveillance system, will assess whether the system is serving a useful purpose while meeting the surveillance system's overall objectives. 


\section{References}

Ahmed, A. H., Nicholson, K. G., Nguyen-van Tam, J. S., Pearson, J. C. (1997). Effectiveness of influenza vaccine in reducing hospital admissions during the 1989-90 epidemic. Epidemiology Infection, 118(1), 27-33.

Barker, W. H. \& Mullooly, J. P. (1980). Impact of epidemic type A influenza in a defined adult population. American Journal of Epidemiology, 112(6), 798-811.

Barker, W. H. \& Mullooly, J. P. (1981). Underestimation of the role of influenza and pneumonia in causing excess mortality. American Journal of Public Health, 71(6), 643-645.

Barker, W. H. \& Mullooly, J. P. (1982). A study of excess mortality during influenza epidemics in the United States, 1968-1976. American Journal of Epidemiology, 115(3), 479-480.

Brydak, L. B., Rajkowski, T., \& Machala, M. (1998). Humoral antibody response following influenza vaccination in patients with nephrotic syndrome. Anitinfection Drugs Chemotherapy, 16(2), 151-5.

Buffington, J., Bell, K. M., LaForce, F. M. (1991). A target-based model for increasing influenza immunizations in private practice. Journal of General Internal Medicine, 6, 204-209.

Canadian Communicable Disease Report (2001). Statement on influenza vaccination for the 2001-2002 season. 
Canadian Institute for Health Information (1999). Hospital Morbidity Database. Ottawa.

Centres for Disease Control (2001). Updated guidelines for evaluating surveillance systems. $M M W R, 50(\mathrm{RR} 13), 1-35$.

Centres for Disease Control and Prevention (2001a). Pandemic influenza: a planning guide for state and local officials (Draft 2.1). National Vaccine Program.

Centres for Disease Control and Prevention (1998). Prevention and control of influenza: recommendations of Immunization Practices Advisory Committee. $M M W R_{2}$ 41(RR-9), 1-17.

Chin, J. (2000). Control of communicable disease manual ( $\left.17^{\text {th }} \mathrm{ed}.\right)$. Washington, DC: American Public Health Association.

Committee on the Clinical Trials of Influenza Vaccine (1953). Clinical trials of influenza vaccine. $B M J, 2,1173-1177$.

Demicheli, V., Rivetti, D., Deeks, J. J., \& Jefferson, T. O. (2000). Vaccines for preventing influenza in healthy adults. Cochrane Database System Review, 2, Cd001269.

Descamps-Latscha, B. \& Chatenoud, L. (1996). T cells and B cells in chronic renal failure. Seminars in Nephrology, 16, 183-191.

Dewals, P., Carbonneau, M., \& Payette, H. (1996). Influenza ans pneumococcal vaccine in long term care facilities in two regions of Quebec. Canadian Journal of Infectious Disease, 7, 296-300. 
Diepersloot, R. J., Bouter, K. P. \& Beyer, W. E. (1987). Humoral immune response and delayed hypersensitivity to influenza vaccine in patients with diabetes mellitus. Diabetologia, 30, 397-401.

Duclos, P., Arruda, H., \& Dessau, J. C. (1996). Immunization of non institutionalized adults in Quebec. CCDR, 22, 177-82.

Embree, J. (2001). It is time for a national immunization strategy. Pediatric Infectious Disease Notes, 6(6), 301-302.

Furth, S. L., Neu, A. M. \& McColley, S. A. (1995). Immune response to influenza vaccination in children with renal disease. Pediatric Nephrology, 9(5), 566568.

Govaert, T. M., Sprenger, M. J., Dianant, G. J., Aretz, K., Masurel, N., \& Knotterus, J. A. (1994). Immune response to influenza vaccination of elderly people. A randomized double blind placebo controlled trial. Vaccine, 12(13), 1185-1187.

Grekas, D. \& Alivanis, P. \& Kotzadamis, N. (1992). Influenza vaccination in chronic hemodialysis patients. Renal Failure, 14(4), 575-578.

Green, L. A., Miller, R. S., Reed, F. M., Iverson, D. C., \& Bailey, G. E. (1993). How representative of typical practice are practice-based research networks? A report from ambulatory sentinel practice network inc.(ASPN). Archives of Family Medicine, 2(9), 939-949. 
Gruber, W. C. (1998). Children as target for immunization. In K. G. Nicholson, R. G. Webster, \& A. J. Hay (Eds.), Textbook of influenza (pp. 435-444). Blackwell Science Ltd.

Health Canada (1993). Canadian consensus conference on influenza. Canada Communicable Disease Report, 19(17), 136-146.

Health Canada (2002). Canadian immunization guide. Ottawa.

Health Canada (1998). Economic burden of illness in Canada. Population and Public Health Branch. Ottawa.

Health Canada (2000b). Influenza. Population and Public Health Branch. Ottawa.

Health Canada (2000a). Statement on influenza vaccination for 2000-2001 season. Canada Communicable Disease Report, 26(17), 141-148.

Izurieta, H. S., Thompson, W. W., \& Kramarz, P. (2000). Influenza and the rates of hospitalization for respiratory disease among infants and young children. New England Journal of Medicine, 342, 232-239.

Kilbourne, E. D. (1987). Influenza. New York: Plenum Medical Book Company. Macdonald, J. C., \& Andrews, B. E. (1955). Diagnostic methods in an influenza vaccine trial. $B M J, 2,1232-1235$.

Macdonald, A. J., Roberecki, S. A., and Conway, N. L. (1996). Influenza immunization Surveillance in rural Manitoba. Canadian Journal of Public Health, 87(3), 163-165. 
Manitoba Centre for Health Policy and Information (2001). Influenza's influence: is an early warning system possible.

Manitoba Health (2000). Influenza in Manitoba 1999-2000 season.

McArthur, M. A., Simor, A. E., Campbell, B., \& McGeer, A. (1999). Influenza vaccination in long term care facilities: structuring programs for success. Infection Control and Hospital Epidemiology, 20, 499-503.

Members of the Commission on Influenza (1953). A clinical evaluation of vaccination against influenza. $J A M A, 124,982-985$.

Neuzil, K. M., Hohlbein, C., \& Zhu, Y. (2002). Illness among school children during influenza season: effect on school absenteeism, parental absenteeism from work, and secondary illness in families. Archives of Pediatrics and Adolescent Medicine, 156(10), 986-991.

Neuzil, K. M., Mellen, B. G., Wright, P. F., Mitchel, E. F., \& Griffin, M. R. (2000). New England Journal of Medicine, 342, 225-231.

Nichol K. L. (2001). Cost benefit analysis of a strategy to vaccinate healthy working adults against influenza. Archives of Internal Medicine, 161(5), 749-759.

Nichol, K. L. \& Hauge. M. (1997). Influenza vaccination of health care workers. Infection Control and Hospital Epidemiology, 18, 189-194.

Ontario Ministry of Health and Long Term Care (2001). Universal influenza immunization program. 
Potter, J., Stott, D. J., \& Roberts, M. A. (1999). Influenza vaccination of health care workers in long term care hospitals reduces the mortality of elderly patients. Journal of Infectious Diseases, 175, 1-6.

Russell, M. L. (2001). Influenza vaccination in Alberta long-term care facilities. CMAJ, 164(10), 1423-1427.

Squires, S. G., Macey, J. F., Tam, T. (2001). Progress towards Canadian target coverage rates for influenza and pneumococcal immunizations. Division of Respiratory Diseases, Centre for Infectious Disease Prevention and Control. Ottawa.

Statistics Canada (1996). Age Standardized Mortality Rates. Ottawa..

Statistics Canada (1997). Selected Leading Causes of Death. Ottawa.

Steele, R. W. (1994). Current status of vaccines and immunoglobulins for children with renal disease. Pediatric Nephrology, 8, 7-10.

Tam, T. W. (1999). Preparing for influenza epidemics and pandemics in the new millennium. Canadian Journal of Public Health 90(5), 293-294.

Upshur, R. E., Knight, K., \& Goel, V. (1999). Time series analysis off the relation between influenza virus and hospital admissions of the elderly in Ontario, Canada, for pneumonia, chronic lung disease, and congestive heart failure. American Journal of Epidemiology, 149(1), 85-92.

Wilde, J. A., Mcmillan, J. A., \& Serwint, J. (1999). Effectiveness of influenza vaccine in health care professionals. A randomized trial. $J A M A, 281,908-13$. 
Yassi, A., Murdazk, C., Cheang, M., Tran, N. \& Aoki, F. Y. (1994). Influenza immunization: knowledge, attitude, and behaviour of health care workers. Canadian Journal of Infection Control, 9(4), 103-108.

Zahner, S. J. (1999). Public health nursing and immunization surveillance.

Public Health Nursing, 16(6), 384-389. 


\section{Appendix A}

\section{Logic Model}

Influenza Immunization Surveillance Form ( doses administered / doses distributed)

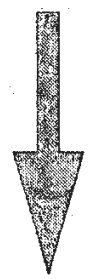

Number of Individuals Vaccinated

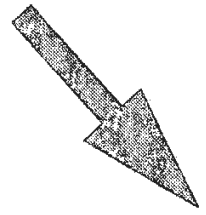

Other Surveys

(Population Health Survey)

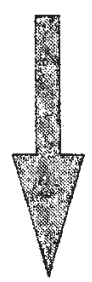

Number of Individuals Eligible for Vaccination

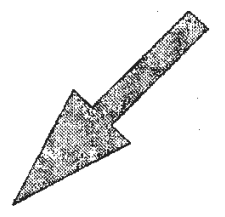

Proportion of Eligible Groups Vaccinated

(Number of Individuals Vaccinated / Number of Eligible Individuals)

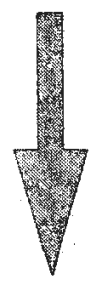

Identification of Target for Influenza Immunization Campaign to Increase Coverage

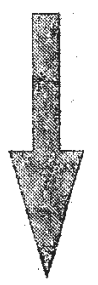

Increased Influenza Vaccination Rates 
Influenza Immunization Surveillance Form

2001 - 2002 Season

\begin{tabular}{|c|c|c|c|c|c|c|c|c|c|c|c|c|c|c|c|}
\hline \multirow{2}{*}{$\begin{array}{c}\text { Date of } \\
\text { momunization } \\
(Y / M / D)\end{array}$} & \multirow{2}{*}{ Gender } & \multirow[b]{2}{*}{$\begin{array}{c}\text { Date of } \\
\text { Birth }\end{array}$} & \multicolumn{9}{|c|}{ Reason for Immunization } & \multirow{2}{*}{\multicolumn{2}{|c|}{$\begin{array}{c}\text { Did patient receive } \\
\text { inlluenza vaccine } \\
\text { last yean? }\end{array}$}} & \multirow{2}{*}{\multicolumn{2}{|c|}{$\begin{array}{l}\text { Cid patient ever receive } \\
\text { pneumococcal vaccine? }\end{array}$}} \\
\hline & & & $\begin{array}{l}\text { Cardiac } \\
\text { Disorder }\end{array}$ & $\begin{array}{l}\text { Pulmonary } \\
\text { Disorder }\end{array}$ & Diabetes & Cancer & Renal Disease & $\begin{array}{l}\text { Resident of Long } \\
\text { Term Care Facilly }\end{array}$ & $\begin{array}{l}\text { Health Care } \\
\text { Stalt }\end{array}$ & Age & Other & & & & \\
\hline & $M \quad F$ & & & & & & & & & & & Yes & No & Yes & No \\
\hline & $M \quad F$ & & & & & & & & & & & Yes & No & Yes & No \\
\hline & $M \quad F$ & & & & & & & & & & & Yes & $\mathrm{No}$ & Yes & No \\
\hline & $M \quad F$ & & & & & & & & & & & Yes & No & Yes & $\mathrm{No}$ \\
\hline & $M \quad F$ & & & & & & & & & & & Yes & No & Yes & No \\
\hline & $M \quad F$ & & & & & & & & & & & Yes & No & Yes & No \\
\hline & $M \quad F$ & & & & & & & & & & & Yes & No & Yes & No \\
\hline & $M \quad F$ & & & & & & & & & & & Yes & No & Yes & No \\
\hline & $M \quad F$ & & & & & & & & & & & Yes & No & Yes & No \\
\hline & $M \quad F$ & & & & & & & & & & & Yes & No & Yes & No \\
\hline & $M \quad F$ & & & & & & & & & & & Yes & No & Yes & No \\
\hline & $M \quad F$ & & & & & & & & & & & Yes & No & Yes & No \\
\hline
\end{tabular}

*Please Check the appropriate box to indicate reason for immunization

Return completed forms to: Dr. Catherine Donovan

Medical Officer of Health

P.O. Box 70

Holyrood, NF AOA 2RO 
Appendix C:
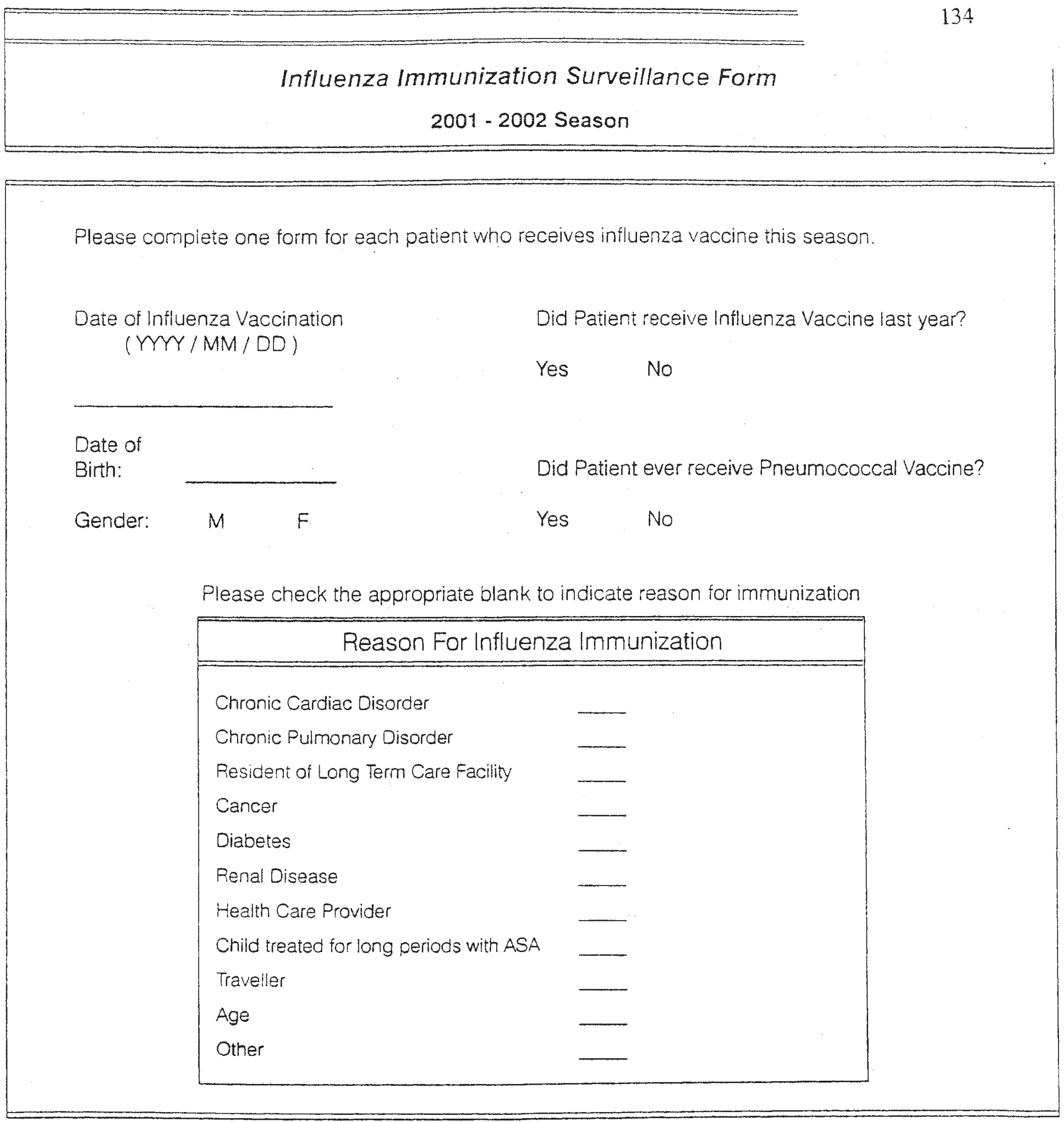

Return completed forms to: Or. Catherine Donovan

Medical Officer of Health

P.O. Box 70

Holyrood, NF

$M \quad 0933$

AOA 2RO 
TALLY SHEET

for recipients of Influenza Vaccine Fall - 2001 Please complete at the end of your clinic day

\begin{tabular}{|c|c|c|}
\hline Date & \# doses for 65 and over & \# doses for those of any age with chronic illness \\
\hline & & \\
\hline & & \\
\hline & & \\
\hline & & \\
\hline & & \\
\hline & & \\
\hline & & \\
\hline & & \\
\hline & & \\
\hline & & \\
\hline & & \\
\hline &. & \\
\hline & & \\
\hline & & \\
\hline & & \\
\hline & & \\
\hline & & \\
\hline & & \\
\hline & & \\
\hline & & \\
\hline & & \\
\hline & & \\
\hline & & \\
\hline & & \\
\hline Total & & \\
\hline
\end{tabular}

When complete please fax to: 
Appendix E: Letter from $\mathrm{MOH}$

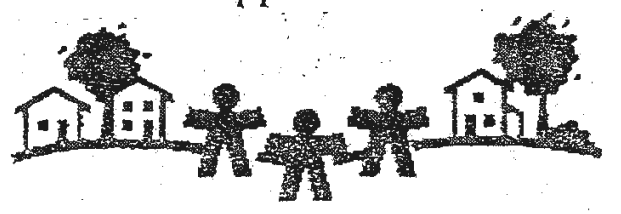

\section{HEALTH AND COMMUNTTY SERVICES \\ EASTERN BOARD \\ Health Promotion/Protection Division \\ P.O. Box 70, Holyrood, NF, AOA 2RO \\ Tel: (709) 229-1573 Fax: (709) 229+1591}

August 7, 2001

\section{Dear Doctor/Nurse:}

Enclosed is information regarding influenza immunization and vaccine order forms for the upcoming influenza season. As an additional feature of the program this year, Eastern is participating in an influenza immunization surveillance project. As you are aware, the region and the province as a whole has little information on the real rates of immunization for influenza, we only know how much vaccine is distributed. As we plan for Pandemic Influenza, it is critical for us to have a better understanding of who is actually being immunized and how successful our efforts are. This is necessary not only to anticipate the impact of pandemic influenza but also to develop targeted promotional campaigns to improve immunization rates and ultimately to decrease the impact of an outbreak. Your collaboration in this effort is essential.

Further information on this special project is enclosed. The project requires the collection of minimal information on the patients to whom you provide vaccine. I am requesting that you return this information to me. Specific directions will accompany your vaccine.

Should you choose not to participate in this project, you will receive your vaccine as usual however your contribution will make it possible for us to design future surveillance activities which are most suitable for you. Your participation is critical to our success.

Thank you in advance for your anticipated cooperation.

Sincerely yours,

Catherine Donovan, M.D., M.H.Sc.

Medical Health Officer

$\mathrm{CD} / \mathrm{sd}$

Encl. 
Appendix F:

\section{Letter of Explanation From Investigator}

Dear Health Care Provider;

I am a Masters student at Memorial University's School of Nursing and I am conducing a research study on influenza immunization surveillance. This study is supported by Eastern Health and Community Services as a part of an influenza immunization initiative. This study is to assess a mechanism of influenza immunization surveillance.

Participation in this study requires that you complete a surveillance form for each patient that receives the influenza vaccine in your clinic/practice. The forms are very simple to complete and will only take a couple of minutes per patient. The forms will then be returned in stamped addressed envelopes provided to you. Participation in this study will give you an opportunity to provide feedback and input into a surveillance system.

For further details on this study please see the attached study explanation. If you have any further questions on this study please feel free to call me at WE will be calling you the week after you receive your vaccine and influenza immunization surveillance forms, and we will send you fax reminders every month unless you notify us otherwise.

Sincerely,

Suzette Porter 


\section{Appendix G:}

\section{Respondent Telephone Interview Questionnaire}

1. Were you able to account for all the influenza vaccines you administered this season by using the influenza immunization surveillance form? Why or why not?

2. Is this the surveillance form you would like to see used if influenza immunization surveillance continues in future seasons? Why or Why not?

3. Who completed the influenza immunization surveillance forms?

4. What is you opinion on influenza immunization surveillance in general? 


\section{Appendix H:}

\section{Non Respondent Telephone Interview Questionnaire}

1. If influenza immunization surveillance continued what changes would you make in the forms or data collection process?

2. What is your opinion on influenza immunization surveillance in general? 


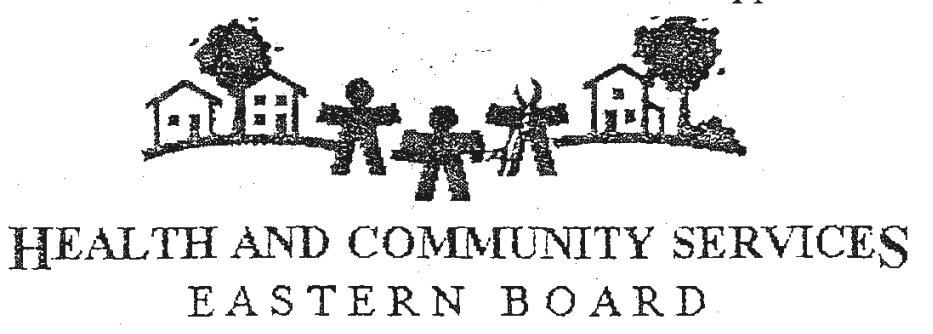

\section{HEALTH AND COMMUNITY SERVTCES \\ EASTERN BOARD}

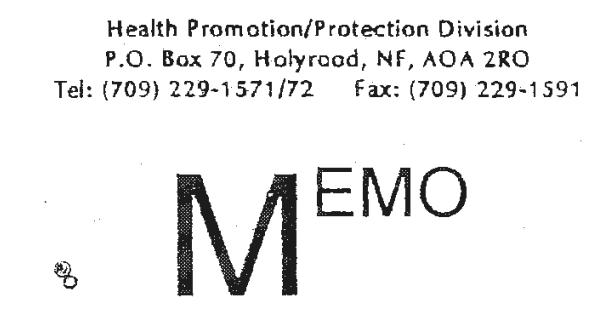

RE: Re: Masters Thesis "Tracking Influenza Immunization in the Community", Suzette Porter

FROM: $\quad$ Catherine Donovan, Medical Health Officer

DATE: $\quad$ August 7, 2001

Health \& Community Services, Eastern Region wishes to express its full support for this project proposal. The results of the project will help to provide information that will be immediately helpful to us in terms of increased knowledge about immunization coverage and will also be useful in planning future surveillance strategies. The project will also provide data of relevance to other regions and the province. This project is very timely in light of current initiatives related to planning for Pandemic Influenza.

This organization will provide in-kind financial contributions through administrative support in addition to promoting the project during our influenza immunization campaign.

Catherine Donovan, M.D. M.H.Sc.

Medical Health Officer

CD/sd 


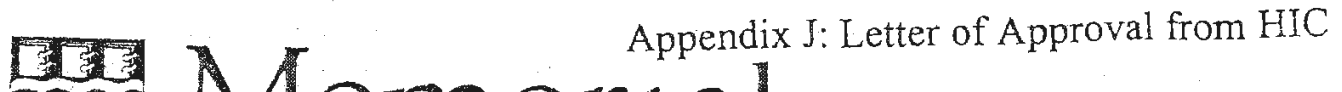

Memorial

University of Newfoundland

Human Investigation Committee

Research and Graduate Studies

Faculty of Medicine

The Health Sciences Centre

August 29, 2001

Reference \#01.146

Ms. Suzette Porter

P.O. Box $304^{4}$

Manuels, NF

AIW 1 M9

Dear Ms. Porter:

At a meeting held on August 23, 2001, your application entitled "Tracking Influenza Immunization in the Community" was reviewed by the Human Irvestigation Committee. The Committee granted approval of the application noting the potential difficulty in receiving a high response rate. A small note was made that Dr. Catherine Donovan is also a supervisor on this thesis study. This was not noted in the application.

Sincerely,

Sharon K. Buehler, PhD

Co-Chair

Human Investigation Committee
Catherine Popadiuk, M.D., F.R.C.S.(C)

Co-Chair

Human Investigation Committee

SKB:CPljglc

C Dr. C. Loomis, Acting Vice-President, Research

Dr. R. Williams, Vice-President, Medical Services, HCC

Dr. D. Moralejo, Supervisor 


